ORNL/TM-2014/652

\title{
Neutron Scattering and High Magnetic
}

\section{Fields}

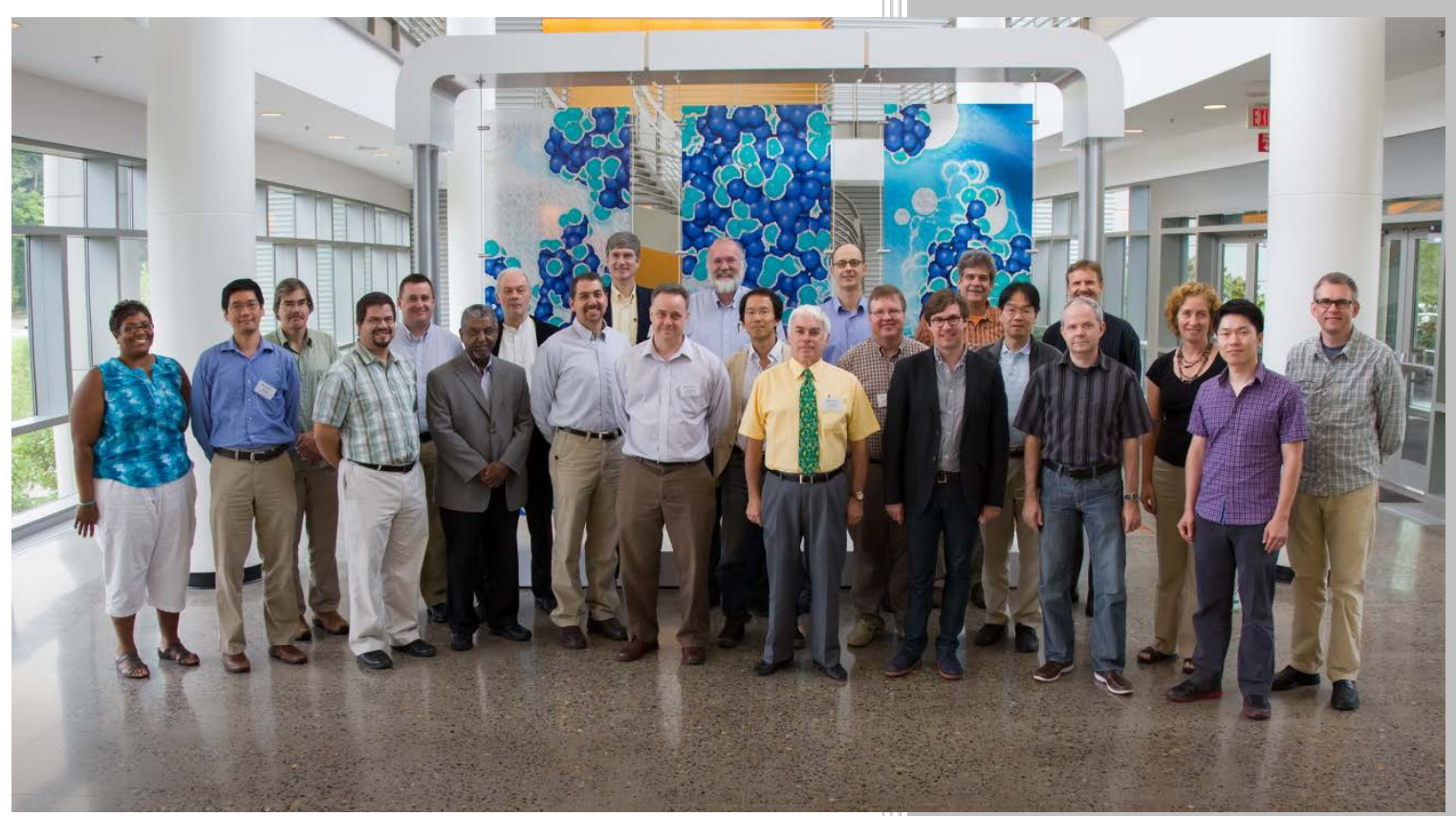

Approved for public release.

Distribution is unlimited.

Matthew B. Stone

Barry L. Winn

Date: November 25, 2014 


\section{DOCUMENT AVAILABILITY}

Reports produced after January 1, 1996, are generally available free via US Department of Energy (DOE) SciTech Connect.

Website http://www.osti.gov/scitech/

Reports produced before January 1, 1996, may be purchased by members of the public from the following source:

National Technical Information Service

5285 Port Royal Road

Springfield, VA 22161

Telephone 703-605-6000 (1-800-553-6847)

TDD 703-487-4639

Fax 703-605-6900

E-mail info@ntis.gov

Website http://www.ntis.gov/help/ordermethods.aspx

Reports are available to DOE employees, DOE contractors, Energy Technology Data Exchange representatives, and International Nuclear Information System representatives from the following source:

Office of Scientific and Technical Information

PO Box 62

Oak Ridge, TN 37831

Telephone 865-576-8401

Fax 865-576-5728

E-mail reports@osti.gov

Website http://www.osti.gov/contact.html

This report was prepared as an account of work sponsored by an agency of the United States Government. Neither the United States Government nor any agency thereof, nor any of their employees, makes any warranty, express or implied, or assumes any legal liability or responsibility for the accuracy, completeness, or usefulness of any information, apparatus, product, or process disclosed, or represents that its use would not infringe privately owned rights. Reference herein to any specific commercial product, process, or service by trade name, trademark, manufacturer, or otherwise, does not necessarily constitute or imply its endorsement, recommendation, or favoring by the United States Government or any agency thereof. The views and opinions of authors expressed herein do not necessarily state or reflect those of the United States Government or any agency thereof. 
Neutron Sciences Directorate

\section{Report from the}

Workshop on Neutron Scattering and High Magnetic Fields

September 4-5, 2013

Matthew B. Stone

Barry L. Winn

Date Published:

November 25, 2014

Prepared by

OAK RIDGE NATIONAL LABORATORY

Oak Ridge, Tennessee 37831-6283

managed by

UT-BATTELLE, LLC

for the

US DEPARTMENT OF ENERGY

under contract DE-AC05-00OR22725 


\section{CONTENTS}

Page

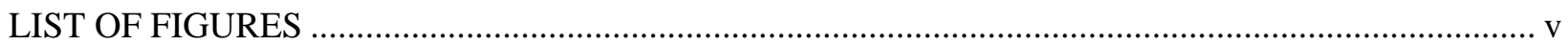

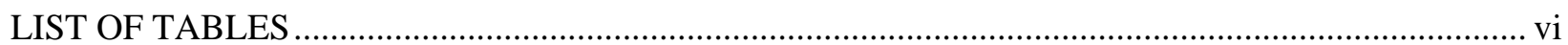

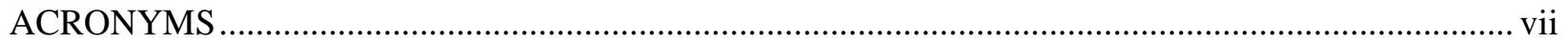

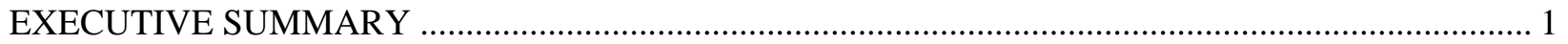

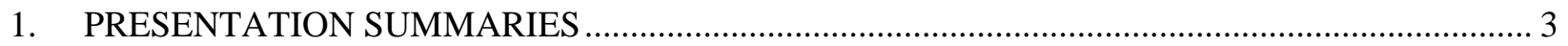

1.1 Alan Tennant, ORNL - 'Welcome and Charge.' '.................................................................... 3

1.2 Stephen Nagler, ORNL - 'Neutron Scattering and Condensed Matter at ORNL.' .................... 3

1.3 Greg Boebinger, National High Magnetic Field Laboratory, Florida State University-

'Probing Quantum Matter: Neutrons and High Magnetic Fields.' ............................................ 4

1.4 Chuck Mielke, National High Magnetic Field Laboratory, Los Alamos National

Laboratory - 'Magnet Technologies for Neutron Scattering.' ................................................... 5

1.5 Gary Lynn, ORNL- 'Magnet Sample Environments at ORNL: Status and Near-Term

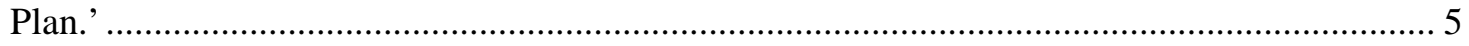

1.6 Peter Smeibidl, Helmholtz Zentrum Berlin- 'High Field Capabilities, Opportunities, and

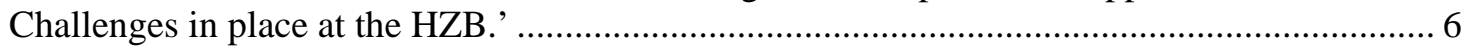

1.7 Yasuo Narumi, Tohoku University- '40 T Pulsed Magnetic Fields for Neutron Diffraction - Recent Progress and Applications.' '................................................................... 7

1.8 Mark Bird, National High Magnetic Field Laboratory, Florida State University- 'DC Magnet Technologies Relevant for Neutron Scattering.' ........................................................ 9

1.9 Kenneth Herwig, ORNL- 'Challenges and Opportunities of the Second Target Station.'........ 10

1.10 Garrett Granroth, ORNL- 'Zeemans: A Dedicated High Field Beam Line for the SNS.' ....... 11

1.11 John Tranquada, Brookhaven National Laboratory- 'Probing Intertwined Orders in Cuprate Superconductors with High Magnetic Fields.'

1.12 Morten Eskildsen, University of Notre Dame- 'Vortex Lattice Metastability and Power Law Dynamics in MgB2.'...

1.13 Martin Greven, University of Minnesota - 'Neutron Scattering Studies of High-Tc

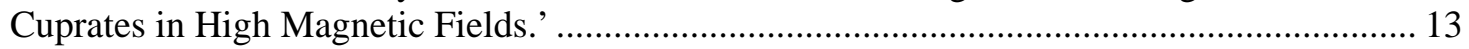

1.14 Dean Myles, ORNL- 'Dynamic Nuclear Polarization and Biology.'....................................... 13

1.15 Valery Kiryukhin, Rutgers University- 'Anisotropic Magnets in High Magnetic Fields.' ...... 15

1.16 Collin Broholm, Johns Hopkins University- 'The high field frontier and quantum

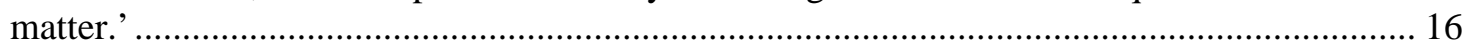

1.17 Meigan Aronson, Brookhaven National Laboratory and Stony Brook University 'Opportunities for High Field Neutron Scattering Experiments in Quantum Critical

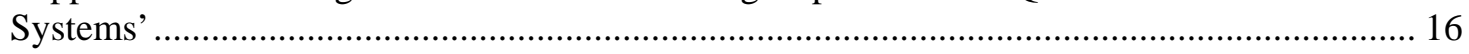

1.18 Young Lee, Massachusetts Institute of Technology-'Scattering Studies of Quantum

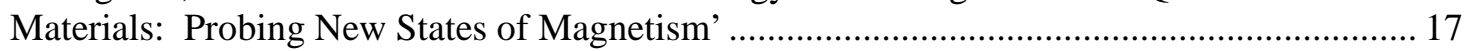

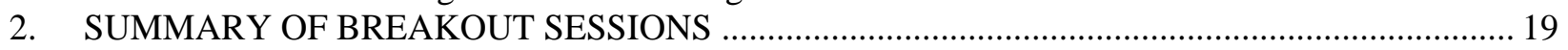

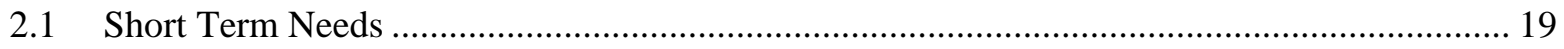

2.2 Strategy to High Magnetic Fields at SNS and HFIR ....................................................... 19

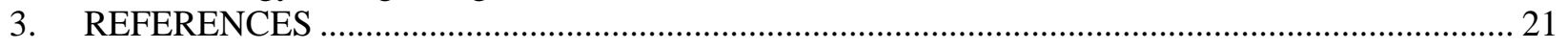

APPENDIX A. PARTICIPANTS, PRESENTATIONS AND AGENDA …........................................ 22

APPENDIX B. MAGNETIC FIELD SAMPLE ENVIRONMENTS AVAILABLE AT NEUTRON

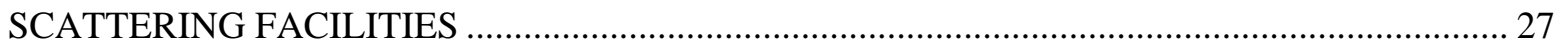




\section{LIST OF FIGURES}

$\begin{array}{lll}\text { Figure } & \text { Page }\end{array}$

Figure 1. Cross section of the HZB hybrid magnet from Ref [4]. ..................................................... 7

Figure 2. Illustration of pulsed field measurement geometry in use at the SEQUOIA time of flight chopper spectrometer instrument at the SNS.

Figure 3. Rendering of the buildings of the Spallation Neutron Source at Oak Ridge National

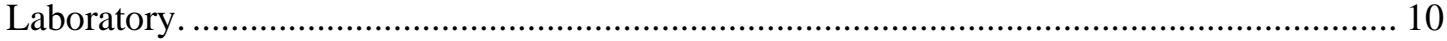

Figure 4. Rendering of a potential instrument layout of the second target station at the SNS. ................ 11

Figure 5. Coherent, incoherent and total scattering cross section of hydrogen as a function of the proton polarization for fully polarized neutrons. ................................................................... 14

Figure 6. Total magnetic field sample environment capability available at several neutron scattering facilities. 


\section{LIST OF TABLES}

Table

Table 1. Sample environments with high magnetic field and low temperatures available at the

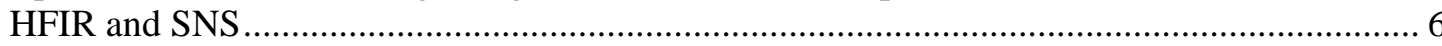

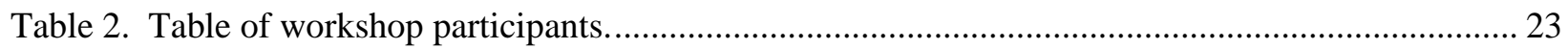

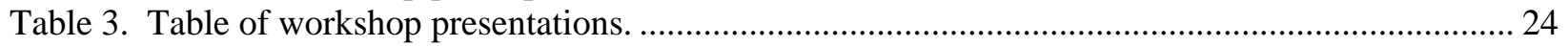

Table 4. List of neutron scattering facilities worldwide with a user program and operation of magnetic field sample environments...................................................................................... 27 


\section{ACRONYMS}

\begin{tabular}{|c|c|}
\hline 1D & One Dimensional \\
\hline $2 \mathrm{D}$ & Two Dimensional \\
\hline ARPA-E & Advanced Research Projects Agency-Energy \\
\hline BSCCO & Bismuth strontium calcium copper oxide \\
\hline DC & Direct Current \\
\hline DNA & Deoxyribonucleic acid \\
\hline DNP & Dynamic Nuclear Polarization \\
\hline DOE & Department of Energy \\
\hline EPR & Electron Paramagnetic Resonance \\
\hline HFIR & High Flux Isotope Reactor \\
\hline HTS & High Tc Superconducting \\
\hline $\mathrm{Hz}$ & Hertz \\
\hline HZB & Helmholtz Zentrum Berlin \\
\hline ILL & Institut Laue-Langevin \\
\hline ISIS & Name of the spallation neutron source at Rutherford Appleton Laboratory in the UK. \\
\hline J-PARC & Japan Proton Accelerator Research Complex \\
\hline LBCO & Lanthanum barium copper oxide \\
\hline LTS & Low Tc Superconducting \\
\hline $\mathrm{mK}$ & milliKelvin \\
\hline MW & Megawatt \\
\hline NAS & National Academy of Sciences \\
\hline NHMFL & National High Magnetic Field Laboratory \\
\hline NScD & Neutron Scattering Sciences Directorate \\
\hline NSF & National Science Foundation \\
\hline ORNL & Oak Ridge National Laboratory \\
\hline QCMD & Quantum Condensed Matter Division \\
\hline QCP & Quantum Critical Point \\
\hline QSL & Quantum Spin Liquid \\
\hline $\mathrm{R} \& \mathrm{D}$ & Research and Development \\
\hline REACT & Rare Earth Alternatives in Critical Technologies \\
\hline SNS & Spallation Neutron Source \\
\hline SSL & Shastry-Sutherland Lattice \\
\hline STS & Second Target Station \\
\hline $\mathrm{T}$ & Tesla \\
\hline YBCO & Yttrium barium copper oxide \\
\hline
\end{tabular}




\section{EXECUTIVE SUMMARY}

The workshop "Neutron Scattering and High Magnetic Fields" was held September 4-5, 2014 at the Oak Ridge National Laboratory (ORNL). The workshop was held in response to a recent report by the National Research Council of the National Academy of Sciences entitled "High Magnetic Field Science and Its Application in the United States: Current Status and Future Directions."1 This report highlights the fact that neutron scattering measurements carried out in high magnetic fields provide important opportunities for new science. The workshop explored the range of the scientific discoveries that could be enabled with neutron scattering measurements at high fields (25 Tesla or larger), the various technologies that might be utilized to build specialized instruments and sample environment equipment to enable this research at ORNL, and possible routes to funding and constructing these facilities and portable high field sample environments.

Participants included principal investigators from U.S. national laboratories and universities. Technical experts were present from the National High Magnetic Field Laboratory (NHMFL) and international facilities. Technical experts and principal investigators from ORNL were also present. Invited speakers on the first day of the workshop focused upon the techniques and challenges associated with neutron scattering measurements performed in high magnetic fields. The invited speakers on the second day focused primarily upon neutron scattering measurements at high magnetic field and important measurements that would be possible with large magnetic field sample environments at the Spallation Neutron Source (SNS) and the High Flux Isotope Reactor (HFIR) at ORNL.

Recommendations to be made to the Neutron Sciences Directorate at ORNL were solicited on both days of the workshop in a town-hall discussion format. While we provide further detail regarding the workshop in later sections of this report, we summarize here the conclusions of these discussions.

- The current operable magnetic field sample environments at the SNS and HFIR do not provide adequate magnetic field strengths to be internationally competitive. The current state of the art high direct current (DC, i.e. not pulsed) magnetic field sample environment for neutron scattering is between 15 and 17 Tesla. Such sample environments are available from commercial vendors and do not require any research or development on the part of the facilities. The current largest operable magnetic field sample environment available at the SNS is a 5 Tesla vertical field magnet. The current largest magnetic fields available at the HFIR are 4.5 Tesla horizontal and 11 Tesla vertical fields. At least one 15 to 17 Tesla split coil vertical field symmetric magnet should be obtained for use at the SNS immediately. An 11 Tesla horizontal field magnet should be purchased for use at the HFIR immediately pending the success of the imminent design review. In order to be competitive and to able to carry out the best science, the ORNL neutron scattering facilities must upgrade their magnetic field sample environments.

- A high magnetic field (40 Tesla or greater) beamline should be considered to be integrated directly into the design and construction of the second target station (STS) at the SNS. This beamline, currently referred to as Zeemans, would be a versatile neutron scattering instrument built around a large horizontal magnetic field sample environment. The current largest magnetic field neutron scattering beamline is built and is currently being commissioned at the Helmholtz Zentrum Berlin (HZB) facility. The magnetic fields available will be between 25 and 30 Tesla. A 40 to 60 Tesla Zeemans instrument at the SNS will enable the next generation of neutron scattering experiments at high fields to be performed. Decisions will need to be made during the planning phases of the STS regarding the magnet technology to be used in the Zeemans high field magnet. These choices will be primarily based on test results on a high temperature superconducting magnet system under construction at the NHMFL.

- Local expertise in the operation, maintenance and development of magnetic field sample environments needs to be established as soon as possible for the ORNL neutron scattering 
facilities. This will ensure that the magnetic field sample environments remain operational for many years. The operation and maintenance of the Zeemans instrument will also require a group of highly qualified staff.

- ORNL and the NHMFL would benefit from collaborating on specific projects to develop high magnetic field sample environments for use with neutron scattering measurement techniques. This collaboration may involve the establishment of a NHMFL staff and laboratory presence at ORNL. Projects should be strategically chosen to contribute to the development of the Zeemans instrument. These projects may include development and support of a 30 Tesla or greater high repetition rate pulsed magnet and/or a 25 Tesla DC magnet.

- Engagement of US universities is extremely important in developing a suite of magnetic field sample environments at the ORNL neutron scattering facilities. This engagement includes avenues for training and education, and continued feedback concerning ORNL's progress towards these goals.

In the remaining sections of the report, we provide summaries of the individual presentations made by the invited speakers. We then provide a summary of each of the discussion sessions held during the workshop. We also provide several appendices listing the attendees, the speakers, the agenda, and the status of magnetic field sample environments at ORNL and other neutron scattering facilities. 


\section{PRESENTATION SUMMARIES}

\subsection{ALAN TENNANT, ORNL - 'WELCOME AND CHARGE.'}

Dr. Tennant, Chief Scientist, Neutron Scattering Sciences Directorate (NScD), welcomed the presenters and attendees, and charged the attendees to develop an aggressive strategy that integrates high magnetic fields and neutron scattering. This strategy must address what ORNL neutron scattering facilities and strategic partners require for extreme magnetic fields both now and within the next 10 years. Both the 2013 National Academy of Science (NAS) Study on "High Magnetic Field Science and Its Application in the United States"1 and the Workshop on Quantum Condensed Matter at Berkley ${ }^{2}$ in 2013 emphasized the need to couple high fields with neutron scattering measurements in quantum condensed matter research. Another tremendous scientific opportunity at ORNL is the improved signal to noise ratio possible using high magnetic fields to perform dynamic nuclear polarization of hydrogen in biological samples such as protein crystals, providing further information on the structure and function of proteins. In response, this workshop brings together a broad range of technical and scientific expertise in order to explore the scientific potential and needs of the community, and the technological state of the art. This is an active time, because new technologies are making magnets possible that take us into new areas of science, and because of opportunities available due to new ideas for neutron scattering instrumentation, especially the recent developments for the Second Target Station. It is time to push the limits, and take advantage of the very best scientific and technological opportunities.

\subsection{STEPHEN NAGLER, ORNL - 'NEUTRON SCATTERING AND CONDENSED MATTER AT ORNL.'}

Dr. Nagler, division director of the Quantum Condensed Matter Division (QCMD), provided a historical background concerning the neutron scattering facilities and neutron scattering measurements at ORNL for the past seven decades. The current facilities for neutron scattering at ORNL and instrumentation available were described. ORNL hosts the HFIR and SNS neutron sources. The SNS is the world's most intense pulsed neutron source, and the HFIR is one of the world's highest flux reactor based neutron sources. There are 12 instruments in the user program at HFIR and 16 instruments in the user program at SNS. 75\% of the available time at these instruments is in the user program.

Dr. Nagler also discussed the instruments that are operated by the QCMD. The QCMD is responsible for 13 instruments at the HFIR and SNS. These include diffractometers and inelastic instruments. Recently, individual publications have more often presented measurements from multiple instruments from both the SNS and HFIR. The science being pursued by these instruments includes magnetism, multiferroics, unconventional superconductors, quantum magnets, giant magnetoresistive and collosal magnetoresistive materials, superconductors, topological insulators, crystal field excitations and anharmonic lattice dynamics.

A workshop ${ }^{2}$ at the University of Berkley was held in December of 2013 to discuss the status and future of condensed matter and how it relates to the SNS and HFIR at ORNL. Some of the findings of this workshop were discussed by Dr. Nagler. This workshop found that new science is often materials driven and that computation is making greater contributions to the field. It was also noted that extreme sample environments coupled with high flux neutron sources and modern instrumentation are key enablers of new and ground breaking science at neutron scattering facilities. Some of the challenges facing the SNS and HFIR include the continual improvement of instruments, engaging the community to determine their scientific needs, adding capabilities such as high magnetic field sample environments, and developing the instruments and facilities of the future such as the second target station at SNS. 


\subsection{GREG BOEBINGER, NATIONAL HIGH MAGNETIC FIELD LABORATORY, FLORIDA STATE UNIVERSITY- 'PROBING QUANTUM MATTER: NEUTRONS AND HIGH MAGNETIC FIELDS.’}

Dr. Boebinger, Director and Principal Investigator at the National High Magnetic Field Laboratory, gave an overview of the relevance of high magnetic fields to probing quantum matter, and a look at new magnet technologies that are relevant to neutron scattering in particular. Much of the context was set by the recent NAS Report. ${ }^{1}$ A specific recommendation of this report was "New types of magnets should be developed and implemented that will enable the broadest possible range of X-ray and neutron scattering measurements in fields in excess of $30 \mathrm{~T}$ ”. The presentation consisted of a discussion of science on quantum materials enabled by high magnetic fields followed by an overview of magnet technologies which are relevant to neutron sciences.

Quantum materials were identified as one of the key science drivers for the NHMFL. Several examples of topical problems were presented where high magnetic field research has made significant impact. These include studies of unconventional superconductors including exploring the phase diagram of the hidden order material $\mathrm{URu}_{2} \mathrm{Si}_{2}$; quantum spin dimer systems with rich field induced phases such as $\mathrm{SrCu}_{2}\left(\mathrm{BO}_{3}\right)_{2}$ and $\mathrm{Ba}_{3} \mathrm{Mn}_{2} \mathrm{O}_{8}$; frustrated magnets; iridates; multiferroics such as $\mathrm{Ca}_{3} \mathrm{CoMnO}_{6}$ and $\mathrm{CuCrO}_{2}$; anisotropic nanocomposite magnets; and exchange coupled multilayers. All of the presented scientific problems and many of the specific materials have also been studied extensively with both elastic and inelastic neutron scattering techniques. Despite the clear scientific overlap between neutron scattering and high magnetic field research, currently, neutron scattering experiments in continuous magnet fields are restricted to $\sim 15 \mathrm{~T}$. Consequently, large regions of the phase diagrams of materials such as those described above remain unexplored with neutron techniques and expansion of the field range accessible to neutron scattering, as recommended in the NAS report, will have a tremendous scientific payoff.

There have been significant advances in both steady state and pulsed magnet technology which hold great promise for neutron scattering studies under extreme conditions. In the second portion of the talk, an overview of these technologies was presented with particular focus on what could be used for neutron scattering applications. The basic technology options consist of:

- DC Resistive magnets can provide 35 T (20 MW power) for a simple solenoid. Neutron scattering applications often require a split magnet yielding a $20-50 \%$ reduction in peak field.

- The Helmholtz-Zentrum Berlin (HZB) magnet is a DC Hybrid magnet with a superconducting outsert and a resistive insert. Sample access is accommodated through a conical bore allowing for 25-30 T with 4-8 MW power consumption.

- High $\mathrm{T}_{\mathrm{c}}$ superconducting (HTS) magnets are now feasible and yield the promise for much higher fields than conventional superconducting magnets. Concepts being considered for the European Spallation Source include a $25 \mathrm{~T}$ split magnet and a $30 \mathrm{~T}$ conical magnet. These magnets have reduced operating expenses compared to resistive magnets, but considerable $R \& D$ remains and the relevant time frame is 7-10 years. A 32T HTS solenoid under construction at the NHMFL should clarify the role of such magnets for future neutron scattering applications.

- The combination of pulsed magnets and neutron scattering has been used successfully at both SNS and Institut Laue-Langevin (ILL) by the group of Prof. Nojiri. An expanded effort in pulsed fields is the most promising route to achieve 40-60 T. However, the magnet pulse rate will only be every few minutes limiting the scientific applicability of this approach.

Clearly, there are multiple technical options to generate fields in excess of $30 \mathrm{~T}$ for neutron scattering applications as recommended by the NAS report. A DC Hybrid magnet (similar to the HZB magnet) provides the most rapid path to enabling $30 \mathrm{~T}$ with neutron scattering in the United States. HTS magnets may well be the future of such applications but their deployment is a number of years away. The need for such capabilities is clear and community engagement is required to help define the roadmap for magnets as applied to neutron scattering techniques. 


\subsection{CHUCK MIELKE, NATIONAL HIGH MAGNETIC FIELD LABORATORY, LOS ALAMOS NATIONAL LABORATORY - 'MAGNET TECHNOLOGIES FOR NEUTRON SCATTERING.'}

Dr. Mielke discussed the state of the art for pulsed magnets at the NHMFL. The pulsed magnets in use at the NHMFL include a $100 \mathrm{~T}$ pulsed magnet, a $300 \mathrm{~T}$ single shot pulsed magnet, a $60 \mathrm{~T}$ controlled wave form pulsed magnet, and a $65 \mathrm{~T}$ millisecond pulsed magnet. The $65 \mathrm{~T}$ pulsed magnet is the work horse pulsed magnet for the NHMFL. The success of the pulsed magnet user program is also due to standard operation at temperatures as low as $350 \mathrm{mK}$ and the quality of measurement instrumentation available to the user community.

The forces on the current carrying wires in pulsed magnets due to the generated magnetic field need to be accounted for in a magnet's design. The fatigue limit for pulsed magnets due to the tensile stress in the wires is often between 500 and 3000 pulses. Larger systems can be designed for a lifetime of 20000 pulses. The other design consideration for pulsed magnets is their cooling. Liquid nitrogen or water is often used to cool the magnet coils between pulses. Performance of a pulsed magnet is based upon an optimization of strength, ductility and conductivity of the components.

In preparing a pulsed magnet for neutron scattering measurements, one must keep the sample dimensions small (10 $\mu \mathrm{m}$ to $1 \mathrm{~mm})$ to reduce eddy current heating if the sample is a conductor. The repetition rate must also be considered. Experiments will be able to gather more counting statistics if more pulses can be measured. Current repetition rates vary between five and thirty minutes between pulses.

Dr. Mielke also discussed the challenges of operating a successful pulsed magnet sample environment with a neutron scattering instrument. For such measurements to be successful, one must have a high flux neutron source, the ability to narrow beam size for small sample dimensions, and high speed detectors. Currently, pulsed magnets are used for neutron diffraction measurements. An ideal pulsed magnet for inelastic neutron scattering measurements would have a repetition rate of at least $0.5 \mathrm{~Hz}$, the option of a split coil arrangement, and more than 100,000 pulses available.

\subsection{GARY LYNN, ORNL- 'MAGNET SAMPLE ENVIRONMENTS AT ORNL: STATUS AND NEAR-TERM PLAN.’}

Dr. Lynn, leader of the Sample Environment Group, Instrument and Source Division, NScD, presented the current status and immanent capabilities for high magnetic fields and low temperatures, at both the High Flux Isotope Reactor and the Spallation Neutron Source. These are summarized in Table 1.

He requested feedback on whether it would be prudent to replace the $5 \mathrm{~T}$ vertical bore magnet at HFIR nearing end-of-life. The $16 \mathrm{~T}$ vertical bore split coil magnet known as FAT SAM may no longer be functional. A plan for further testing and determination of whether to repair FAT SAM is being developed now.

The sample environment group needs to further develop in-house expertise in magnet environments. Typically the group works with scientists in the ORNL Neutron Sciences Directorate to develop specifications and requirements for purchasing magnets for neutron scattering from various vendors. These superconducting magnets range from $5 \mathrm{~T}$ to $16 \mathrm{~T}$. The sample environment group has some experience with operating these magnets that come from companies like Oxford Instruments and Bruker. When problems arise with the magnets, the group has limited expertise to diagnose and trouble-shoot. While the group should not get into the business of designing new high-field superconducting magnets, they do need to develop experience in diagnosing problems related to operations. 
Table 1. Sample environments with high magnetic field and low temperatures available at the HFIR and SNS. The $16 \mathrm{~T}$ magnet at the SNS is currently not operating and is being triaged for possible repair.

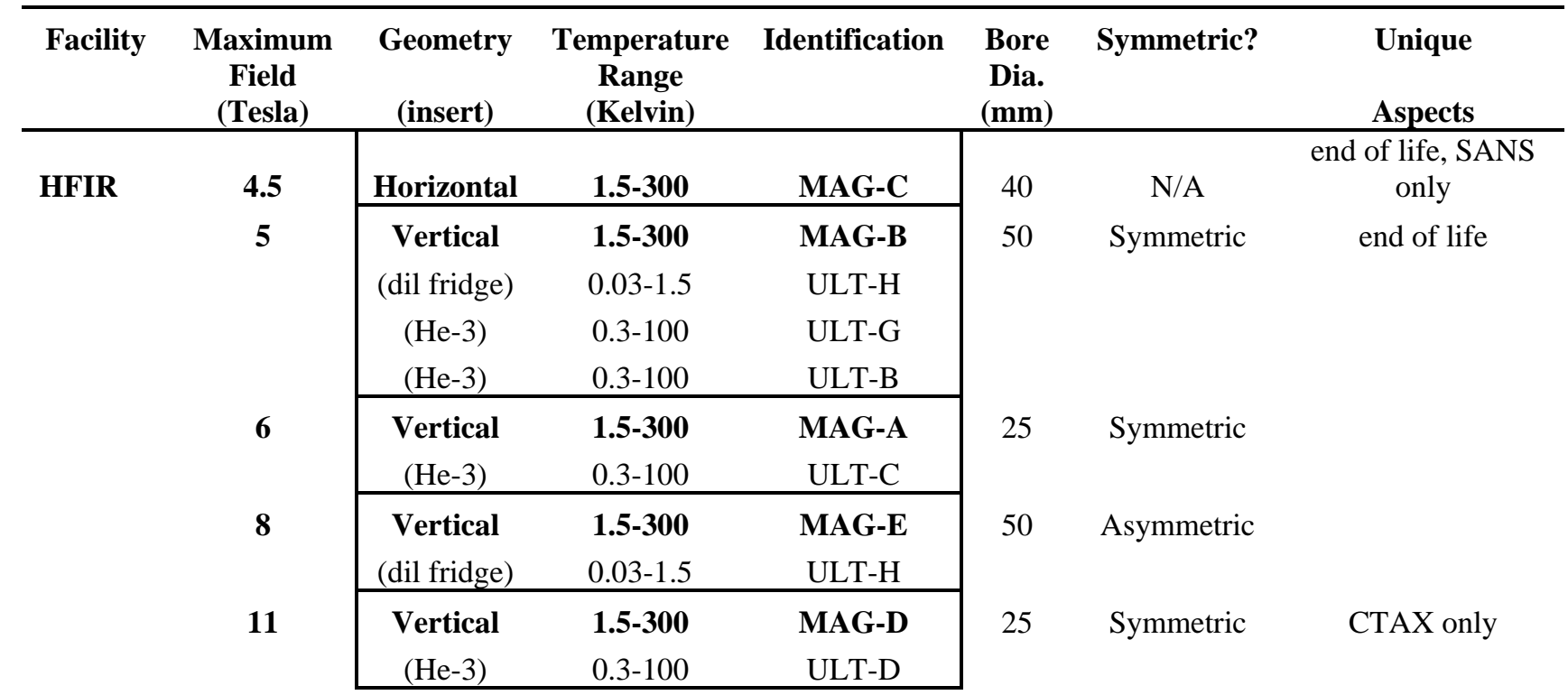

HFIR in design phase

\begin{tabular}{|c|c|c|c|c|c|c|}
\hline 11 & \multicolumn{3}{|c|}{$\begin{array}{ll}\text { Horizontal } & 1.5-300 \\
\end{array}$} & \multicolumn{2}{|c|}{ N/A } & Design phase \\
\hline $1.2-3$ & Vertical & 4-300 & MAG-003 & N/A & & $\begin{array}{l}\text { 50-10 mm gap, } \\
\text { M. Refl. Only }\end{array}$ \\
\hline 5 & Vertical & $1.5-300$ & MAG-001 & 34 & Asymmetric & actively shielded \\
\hline & (dil fridge) & $0.03-1.5$ & ULT-002 & & & \\
\hline pulsed 30 & Horizontal & $1.5-300$ & MAG-006 & & N/A & 5 minute recovery \\
\hline \multicolumn{7}{|l|}{ SNS in procurement } \\
\hline 8 & $\begin{array}{c}\text { Vertical } \\
\text { (dil fridge) }\end{array}$ & $\begin{array}{l}\mathbf{1 . 5}-\mathbf{3 0 0} \\
0.03-1.5\end{array}$ & & 34 & Symmetric & $\begin{array}{l}\text { Wedge supports, } \\
\text { actively shielded }\end{array}$ \\
\hline \multicolumn{7}{|l|}{ SNS magnet triage } \\
\hline 16 & $\begin{array}{l}\text { Vertical } \\
\text { (dil fridge) }\end{array}$ & $\begin{array}{l}\mathbf{1 . 5}-\mathbf{3 0 0} \\
0.03-1.5\end{array}$ & $\begin{array}{c}\text { N/A } \\
\text { ULT-002 }\end{array}$ & 34 & Asymmetric & $\begin{array}{l}\text { Not operating } \\
\text { evaluating repairs }\end{array}$ \\
\hline
\end{tabular}

\subsection{PETER SMEIBIDL, HELMHOLTZ ZENTRUM BERLIN- 'HIGH FIELD CAPABILITIES, OPPORTUNITIES, AND CHALLENGES IN PLACE AT THE HZB.’}

The HZB (formerly the Hahn Meitner Institute) has a 10 MW reactor based neutron scattering facility. In the mid 1990's, HZB decided to be a world leader in hosting high magnetic field sample environments for neutron scattering measurements. In 1997 and 1998 two 15 T superconducting vertical field magnet systems were delivered from Oxford Instruments. At that time, such a high field capability was unique among neutron scattering facilities, and the magnets were in high demand for the user program. Low temperature capabilities and ferromagnetic pole pieces were added to expand the range of temperatures and increase the range of magnetic fields respectively. 
In 2007, the HZB undertook a project with the NHMFL to build a high field magnet for neutron scattering with magnetic fields between 25 and $30 \mathrm{~T}^{3}$ The magnet is a 'series-connected hybrid magnet system' and will initially operate up to $25 \mathrm{~T}$ with a $4 \mathrm{MW}$ resistive copper coil. This coil can later be replaced to operate at $30 \mathrm{~T}$ with an $8 \mathrm{MW}$ system. ${ }^{4}$ The high field magnet is a horizontal field magnet located at an end station of one of the cold neutron guides in the second guide hall of the HZB reactor. Dr. Smeibidl noted that using a 'test-bed' to simulate the magnet load was extremely useful in testing the power supplies and infrastructure that will be used with the final magnet. Initial testing of the high field magnet performance is being done away from its final location at the beamline, to facilitate access. The project required seven years and seven full time employees. There was a cost of $\$ 30 \mathrm{M}$ for the construction of the infrastructure and magnet. Half of the budget was used for cooling and magnet power supplies. It is expected to cost approximately $\$ 1 \mathrm{M}$ per year to operate.

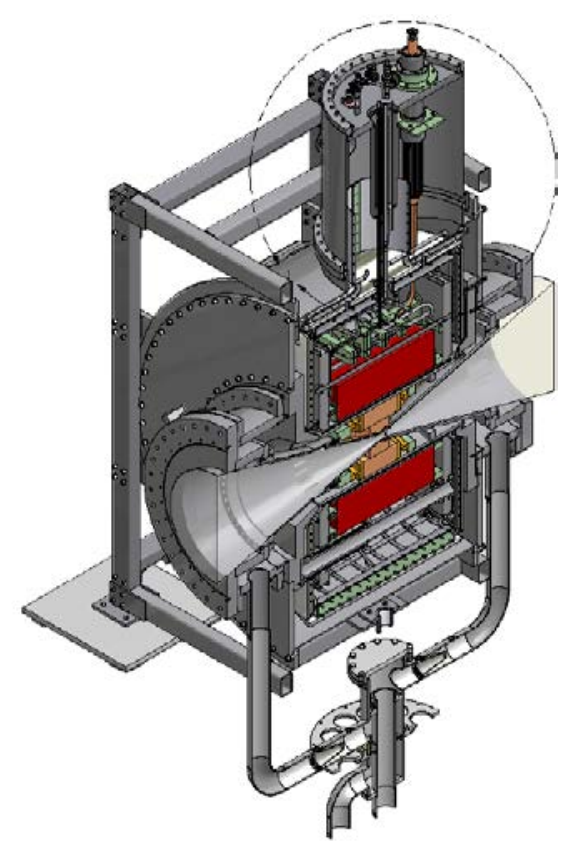

Figure 1. Cross section of the HZB hybrid magnet from Ref [4]. The bore of the magnet is along the same direction as the incident neutron beam. SC coils are shown in red surrounding the inner resistive coils shown in orange.

The philosophy of the high magnetic field sample environment group at HZB has always included a focus on 24/7 support of user experiments. In addition, this group often focuses upon tailored solutions for complex experiments. The staffing profile of this group has always included physicists, technicians and other scientists. For large projects, such as the high field magnet, individual group members would be responsible for individual project components throughout the lifetime of the project. Only the sample handling (sample exchange, sample rotation) and the development and fabrication of the sample cryostat were in-house activities.

\subsection{YASUO NARUMI, TOHOKU UNIVERSITY - ‘ 40 T PULSED MAGNETIC FIELDS FOR NEUTRON DIFFRACTION - RECENT PROGRESS AND APPLICATIONS.’}

Dr. Narumi of the Institute for Materials Research, Tohoku University, began by emphasizing that the scientific motivation for pulsed magnets is the desire to determine complex magnetic structure at new phases that can only be reached at high magnetic fields, in strongly correlated systems such as frustrated antiferromagnets. Questions that have already been or can be addressed using pulsed magnets include the nature of the memory effect in the multiferroic $\mathrm{MnWO}_{4},{ }^{5}$ and the structure of the multiple phases that 
occur around $36 \mathrm{~T}$ in $\mathrm{URu}_{2} \mathrm{Si}_{2}$. The latter system has a critical field $\mathrm{H}_{\mathrm{c}} \sim 26 \mathrm{~T}$ and data acquisition only required $\sim 100$ shots.

The current maximum field strength for DC magnets is limited to $\sim 17 \mathrm{~T}$ for commercially available low $\mathrm{T}_{\mathrm{c}}$ superconductor (LTS) magnets, possibly $25 \mathrm{~T}$ for HTS magnets and up to $35 \mathrm{~T}$ for massive, stationary hybrid LTS / resistive magnet systems, so to reach the new phases that require higher magnetic fields than $17 \mathrm{~T}$ in less than 8 years, or fields higher than $60 \mathrm{~T}$ ever, the only route is via pulsed magnets. Pulsed magnets are classified in several ways: non-destructive vs. destructive, mini-coil vs. buck-up coil, and horizontal bore vs. vertical split-coil. Metrics for these magnets include peak field, time between pulses, time profile of each pulse, typical number of pulses to failure, impact on neighboring instruments, and the size and requirements of the pulsed power supply. The group at the Institute for Materials Research, led by H. Nojiri, is pursuing development and use of both non-destructive mini-coil systems and buck-up coil systems. All coil conductors employ a CuAg alloy wire.

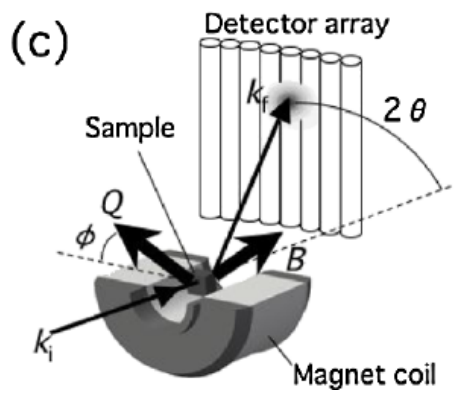

Figure 2. Illustration of pulsed field measurement geometry in use at the SEQUOIA time of flight chopper spectrometer instrument at the SNS. The coil cross-section is shown to illustrate the scattering diagram. $K_{i}$ is the incident neutron wavevector, $K_{f}$ is the scattered neutron wavevector, $B$ is the applied magnetic field direction, $Q$ is the wavevector transfer and $2 \theta$ and $\phi$ are scattering angles. Figure is from Fig.1(c) of Ref [5].

The Nojiri group already has significant experience with horizontal bore mini-coil magnets used with Laue neutron diffraction. Mini-coil systems have the following advantages over buck-up coil systems: they are compatible with existing sample environments, involve modest equipment investment, the coils themselves are "suitcase ready" and easy to transport between neutron scattering facilities, and they require limited pulsed power supply infrastructure and space. Figure 2 illustrates the scattering geometry often used for such mini-coil systems. The current coil system in normal use is a horizontal bore pulsed magnet insert for a liquid helium cryostat, with inner diameter $9.4 \mathrm{~mm}$ and outer diameter $43 \mathrm{~mm}$, with an angle subtended of 26-30 degrees, using a wire cross section of $1 \mathrm{~mm} \times 1.5 \mathrm{~mm}$. The coils are cooled with liquid nitrogen in a separate volume from the sample space. Fields reached at different facilities using these magnets are $30 \mathrm{~T}$ at the SNS (using $25 \mathrm{~kJ}, 5.4 \mathrm{mF}, 5 \mathrm{kV}$ ) ${ }^{3}$. The mini coil cannot go beyond 30 $\mathrm{T}$. The peak field is determined not by the size of power supply, but by the coil itself. With these magnets, the capacitor bank power supplies can be as large as a refrigerator, the magnet is ready for another pulse after about 5 minutes, and the magnet coils last for about 5000 pulses. The time profile for the pulse is dictated by the capacitor bank power supply, and the field intensity varies as a function of incident energy on time of flight diffractometers, so that different phases are measured at different wavelengths during the same magnetic pulse. The same systems have been used at continuous neutron sources like JRR3 and ILL, but it's unlikely that ORNL will develop pulsed magnet systems for HFIR when it has access to a pulsed neutron source with higher peak intensities.

The Nojiri group has also developed a $40 \mathrm{~T}$ and long lifetime buck-up horizontal coil system for beamline 10 at J-PARC. To cool this magnet and the sample, an innovative reversal cryostat was developed that immerses the sample in liquid Helium and the coil in liquid nitrogen. By choosing to cool with liquid nitrogen instead of water, the effective resistance of the coils is reduced significantly, reducing cooling costs per pulse. This system is ready for a new pulse every 14 minutes, uses a wire cross section 
of $2 \mathrm{~mm}$ x $3 \mathrm{~mm}$, and requires $204.8 \mathrm{~kJ}$ at 10mF, $6.4 \mathrm{kV}$ for $40 \mathrm{~T}$. Compact power supplies have been developed for this system outside of Japan, and it has been transported to and demonstrated at ISIS, UK. The transportation costs of both the power supply and the reversal cryostat equipped with the $40 \mathrm{~T}$ coil were approximately $\$ 10,000$. The Nojiri group also supplies the synchrotron facility the Advanced Photon Source with the $40 \mathrm{~T}$ coil to study $\mathrm{TbVB}_{4}{ }^{6}$

Finally, this group is already developing a second generation vertical bore buck-up split coil system, to observe Bragg peaks only visible in the plane perpendicular to the applied magnetic field. The nearterm scientific motivation is to better understand the novel magnetization plateaus of quantum antiferromagnets like $\mathrm{SrCu}_{2}\left(\mathrm{BO}_{3}\right)_{2}$ which require lower temperatures than are achievable with liquid helium cryostats. Therefore an additional requirement for this sample environment system is compatibility with a dilution-refrigerator insert. An additional innovation is to use single crystal sapphire both as a spacer in the split region of the coil and as a near-transparent window for the neutrons; conducting wedges occluding several portions of the plane serve to couple to the lower coils.

\subsection{MARK BIRD, NATIONAL HIGH MAGNETIC FIELD LABORATORY, FLORIDA STATE UNIVERSITY- 'DC MAGNET TECHNOLOGIES RELEVANT FOR NEUTRON SCATTERING.’}

Dr. Bird, Director of the Magnet Science \& Technology Division of the NHMFL, presented options for DC magnet technologies appropriate for use with neutron-scattering techniques. The discussion presented the challenges associated with producing DC magnetic fields up to 30, 35 and $40 \mathrm{~T}$.

The present commercial state-of-the-art vertical-field split DC magnet for neutron-scattering is a $15 \mathrm{~T}$ magnet using Low-Temperature Superconductors (LTS). Such magnets are typically built with aluminum rings to support the split coil. The aluminum rings allow for large beam access (typically $>300^{\circ}$ ), but have a large neutronic background. It is possible to build similar magnets with wedges which occlude certain scattering regions completely, but provide a cleaner background in other regions. However, these magnets typically provide slightly lower field than do the ones with the ring configuration. Magnets providing $14-15$ T exist at HZB, ISIS, ILL and PSI.

A less popular configuration is the horizontal field magnet with conical entrance and exit bores. A commercial LTS version provides $17 \mathrm{~T}$ and is at the University of Birmingham. It travels on occasion to various neutron labs for use.

The NHMFL has been operating a 25 T, 28 MW split coil vertical bore resistive magnet since $2011 .^{7}$ This magnet has optical access, and was not built specifically for a neutron beam line but is used occasionally with an x-ray source. The NHMFL has delivered a horizontal field conical magnet to Helmholtz Zentrum Berlin (HZB) which reached $26 \mathrm{~T}$ in Oct. 2014. It will be installed on the beamline in coming months and should be operational in the $2^{\text {nd }}$ quarter of 2015. The HZB magnet has an LTS outer magnet and a resistive inner magnet and uses $4 \mathrm{MW}$ of dc power. This resistive-superconducting hybrid magnet technology could be scaled up to provide fields as high as $40 \mathrm{~T}$ in the conical configuration.

In recent years a tremendous amount of progress has been made in High-Temperature Superconducting (HTS) materials and magnet technology. The NHMFL is presently developing a $32 \mathrm{~T}$ vertical field superconducting (SC) solenoid which should be the first SC magnet to operate routinely at such a high filed. The magnet consists of an LTS outer section provided by the commercial sector and HTS inner coils developed by the NHMFL using YBCO tape. This magnet is expected to be operational in the $1^{\text {st }}$ quarter of 2016. It appears that a horizontal-field conical-bore magnet providing $30 \mathrm{~T}$ could be developed based on the $32 \mathrm{~T}$ solenoid technology. Furthermore, a vertical-field split magnet providing 25 $\mathrm{T}$ could likely be developed as well. In addition, there has been tremendous progress in both Bi2223 tape and $\mathrm{Bi} 2212$ round-wire which might be appropriate for future magnet systems.

In principle, one can combine HTS, LTS, and resistive magnet technology to produce a hybrid providing $>40 \mathrm{~T}$ dc for neutron scattering. However, there is a tremendous amount of development required to scale up HTS materials to the appropriate scale. 


\subsection{KENNETH HERWIG, ORNL- 'CHALLENGES AND OPPORTUNITIES OF THE SECOND TARGET STATION.’}

Dr. Herwig, leader of the Instrument Projects and Development Group, Instrument and Source Division, NScD, presented the current design concept for the proposed Second Target Station (STS) at the SNS, and the additional opportunities for higher magnetic fields this facility will provide. A rendering of the STS in relation to the current target station is shown in Figure 3. New target and moderator designs are being optimized for short time bursts at $10 \mathrm{~Hz}$, and the highest peak brightness of cold neutrons. This is enabled by a compact, high efficiency target coupled to a compact geometry moderator. Instrument design concepts have been proposed that exploit these strengths of the STS. Elastic-scattering instrument concepts generally leverage the extended bandwidth that comes with the low repetition rate, while inelastic-scattering instrument concepts exploit the low repetition rate via repetition rate multiplication and the significantly increased brightness delivered to the sample. The complementarity of ORNL's three neutron sources will enable strategic positioning of many different kinds of neutron scattering instruments.

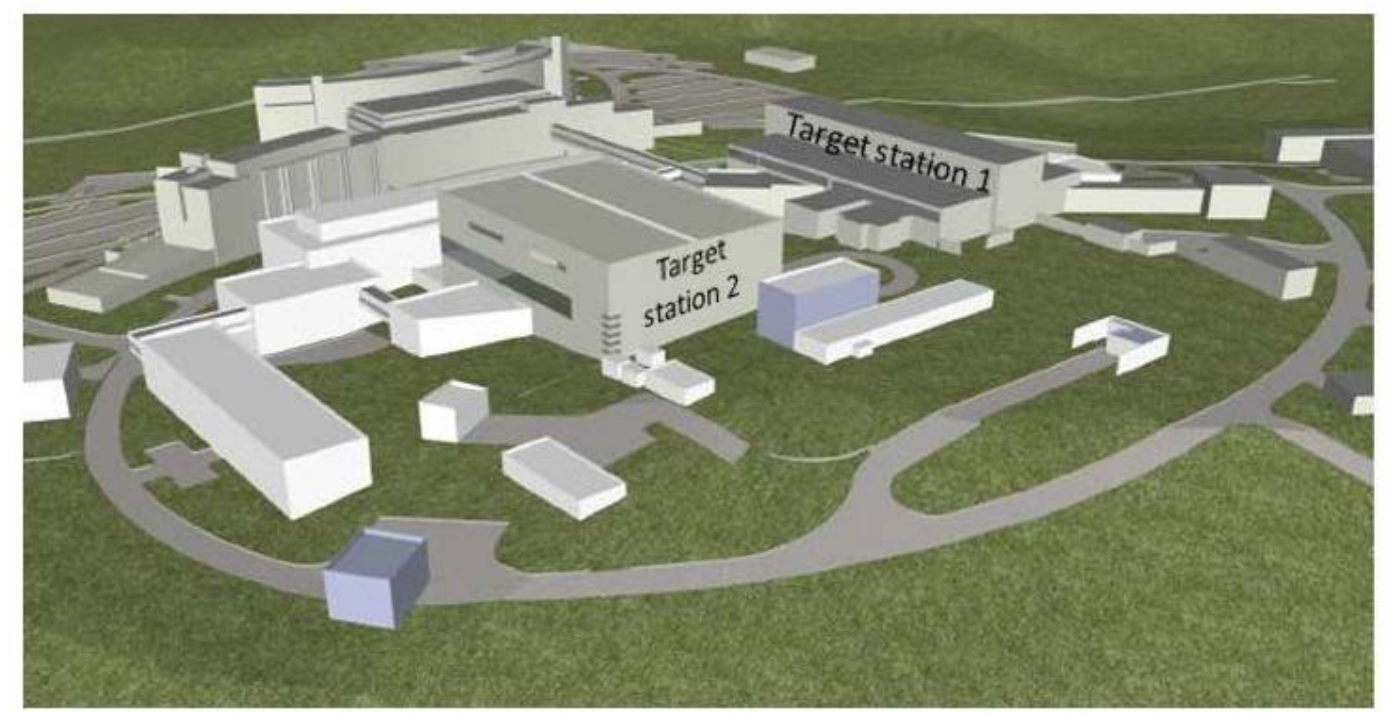

Figure 3. Rendering of the buildings of the Spallation Neutron Source at Oak Ridge National Laboratory. The proposed second target station (STS) is labeled in the figure. White buildings surrounding the STS are proposed support buildings and instrument buildings.

Magnet systems in general will benefit at the STS from the extended bandwidth on the short wavelength end of the spectrum, which can partially compensate for the momentum transfer limitations in scattering angle that superconducting magnet systems impose. Two instruments proposed for the STS will include dedicated high magnetic field systems at sample position: ZEEMANS and DYPOL.

ZEEMANS, described in more detail in the summary for Garrett Granroth's presentation, will benefit from the extended bandwidth that compensates for limited angular acceptance, from higher brightness enabling work with smaller samples, and from the advantage of locating the high-stray-field instrument in the optimum position at a new target building. The proposed location is the northwest corner of the STS, moving ZEEMANS further from field sensitive instruments on the first target station than the previously proposed 14B location of the first target station. A rendering of a potential layout of instruments at the sts is shown in Figure 4. Locating ZEEMANS in this position also orients the magnet axis away from the instruments of the second target station. The ZEEMANS beamline design will be revised to leverage increased brightness, and the opportunity the STS provides to implement repetition-rate multiplication techniques. DYPOL, described in more detail in the summary for Dean Myles' presentation, is a protein crystallography beamline that will benefit from both the extended bandwidth, and the higher brightness 
which will enable examination of much smaller single crystals, closer in size to what is currently expected for synchrotron-based x-ray diffraction.

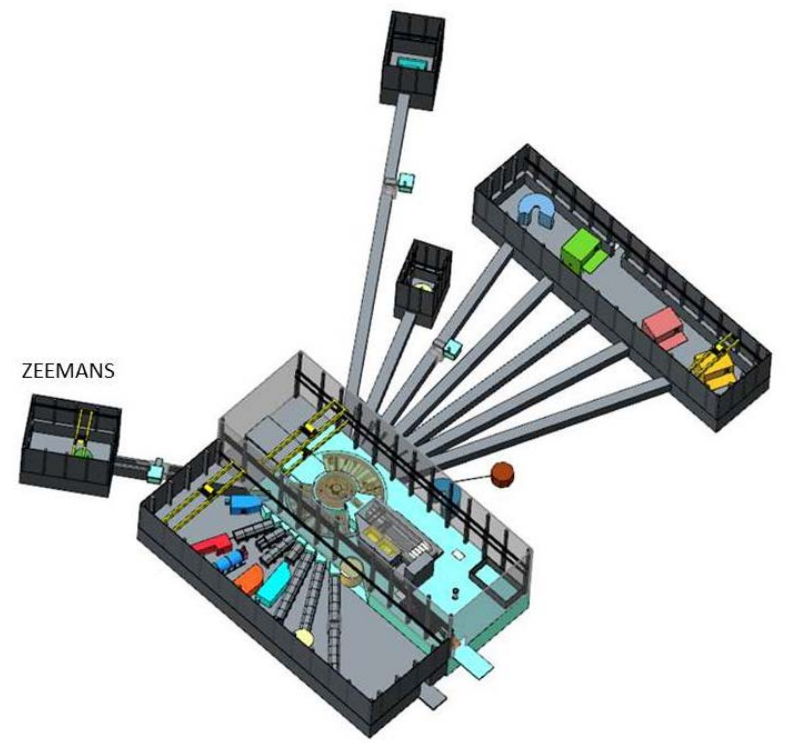

Figure 4. Rendering of a potential instrument layout of the second target station at the SNS. The location of ZEEMANS is indicated in the figure and is further described in the text.

\subsection{GARRETT GRANROTH, ORNL- 'ZEEMANS: A DEDICATED HIGH FIELD BEAM LINE FOR THE SNS.’}

The Zeemans beamline is a proposed dedicated beamline for neutron scattering measurements at high magnetic fields (greater than $30 \mathrm{~T}$ ). This instrument was originally proposed for the first target station at the SNS. It is now being considered for proposal in the instrument suite at the second target station. The configuration of the magnetic field and detectors would allow for spectroscopy, diffraction, SANS and reflectometry measurements.

A Zeemans instrument on the second target station would likely have a flight path from source to sample of between 50 and 60 meters. The neutron guide would be configured with an elliptical vertical cross-section and a curved horizontal section. Repetition rate multiplication would be a viable option to improve counting statistics for inelastic neutron scattering measurements. The detectors would likely be based upon the well-established technology of ${ }^{3} \mathrm{He}$ detectors operating in vacuum as is currently in use at the SNS.

The magnet would likely be a horizontal bore solenoid with a conical cross-section. Cryogenic sample environments for this configuration are not available from vendors. Dr. Granroth suggests watching closely how the high field magnet at HZB is outfitted with low-temperature sample environments. The current design of the Zeemans instrument includes a large rotating differentially pumped seal. This allows for movement of the detectors relative to the horizontal magnetic field. This option will require further design considerations, but is a well-established technology.

\subsection{JOHN TRANQUADA, BROOKHAVEN NATIONAL LABORATORY- 'PROBING INTERTWINED ORDERS IN CUPRATE SUPERCONDUCTORS WITH HIGH MAGNETIC FIELDS.’}

Observing the quantum oscillations in YBCO has required high magnetic fields. These measurements directly probe the Fermi surface of the high temperature superconductor. The specific heat 
measured at high magnetic fields also has oscillatory behavior. This implies that d-wave superconductivity exists to magnetic fields between 25 and potentially as high as $40 \mathrm{~T}$. High magnetic field Hall effect measurements are in disagreement. This mystery could be resolved with high magnetic field neutron scattering measurements.

The superconducting dome in the YBCO phase diagram has been found to split into two separate domes as applied magnetic field increases from 0 to 15 and from 15 to $30 \mathrm{~T}$. This behavior mirrors that of the $\mathrm{LaBaCuO}$ superconductors which have two superconducting domes as a function of hole doping in the phase diagram at zero magnetic field. Neutron diffraction measurements that search for superlattice peaks associated with charge and spin order at high magnetic fields (between 15 and $30 \mathrm{~T}$ ) would be able to conclusively determine the nature of the two superconducting domes in YBCO, and potentially determine a more universal behavior among the high temperature cuprate superconductors.

\subsection{MORTEN ESKILDSEN, UNIVERSITY OF NOTRE DAME- 'VORTEX LATTICE METASTABILITY AND POWER LAW DYNAMICS IN MGB2.’}

When a type-II superconductor is subjected to an applied magnetic field it is threaded by vortices, each carrying one quantum of magnetic flux $\left(\phi_{0}=h / 2 e\right)$, providing a unique probe into the nature of the superconducting state in the host material. In materials with modest or low vortex pinning the vortices, due to their mutual repulsion, arrange themselves in to a regular array - the vortex lattice. The vortex lattice leads to a modulation of the magnetic induction and can therefore be imaged using neutron diffraction. The vortex lattice spacing decreases with increasing applied magnetic field (vortex density) from $144 \mathrm{~nm}$ at $0.1 \mathrm{~T}$ to $14 \mathrm{~nm}$ at $10 \mathrm{~T}$ (typical experimental field values). As such the vortex lattice may be characterized as a large-scale structure and is ideally studied using small angle neutron scattering (SANS).

Neutron scattering studies of the vortex lattice began in the 1970'ies, and include pioneering work at Oak Ridge carried out by Christen and Mook. The use of SANS for such measurements began in earnest following the discovery of high-temperature superconductivity and have since been applied to a wide range of other classes of superconductors. Recent VL SANS studies have been used to provide information about the location and dispersion of gap nodes in $\mathrm{KFe}_{2} \mathrm{As}_{2}$ and $\mathrm{UPt}_{3}$, the field and temperature dependence of the two superconducting bands in $\mathrm{MgB}_{2}$, and intimate details about Pauli limiting and Pauli paramagnetic effects on the vortices in $\mathrm{TmNi}_{2} \mathrm{~B}_{2} \mathrm{C}$ and $\mathrm{CeCoIn}$. In all cases the SANS measurements provided detailed information about the order parameter, thereby constraining theoretical models of superconductivity in the given material.

In this talk Prof. Eskildsen discussed two recent SANS studies from his group at Notre Dame. The first was a novel approach to vortex lattice measurements taking advantage of the transverse field modulation in highly anisotropic superconductors when the field is applied away from a crystalline high symmetry direction. This technique was applied to $\mathrm{Sr}_{2} \mathrm{RuO}_{4}$, commonly considered a paradigm for spintriplet $p$-wave superconductor, and allowed a direct measurement of the intrinsic anisotropy of the superconducting state. The results gave strongly suggest Pauli limiting in this material, raising a serious question about whether equal spin pairing is occurring in this material.

The second topic was the study of novel vortex matter dynamics in the two-band/two-gap superconductor $\mathrm{MgB}_{2}$. In this material long-lived, highly ordered metastable vortex lattice phases are observed. Here it has been possible to use a stop-motion (or stroboscopic) technique to image the vortex lattice as it is driven from the metastable phase to the ground state by successive application of lowamplitude AC magnetic field cycles. This showed a transition characterized by a crossover between multiple power laws, representing an entirely new kind of collective vortex behavior. Furthermore, the results resemble some aspect of jamming in granular materials. Since the vortices are inherent quantum structures, the VL may provide an ideal mono-disperse system for such jamming studies.

The high flux available at the HFIR SANS instruments makes them prime candidates for coupling with high magnetic field sample environments. As new superconducting materials continue to be discovered, there is also a continuous opportunities to provide experimental clues towards a 
comprehensive theory for superconductivity. Empirically, many of the scientifically most interesting materials have very low critical temperatures and/or high upper critical fields. In order to fully explore the superconducting phase diagram in a wide range of superconductors the availability of adequate sample environment is indispensable.

Prof. Eskildsen is involved in determining the specifications of an $11 \mathrm{~T}$ horizontal field superconducting magnet for the SANS at HFIR that would make these beamlines competitive on the world stage for high magnetic field SANS measurements. The applicability for such a magnet will extend far beyond vortex lattice studies, allowing, for instance, studies of magnetic nanoparticles, magnetic inhomogeneities at the nano- and meso-scale, multiferroics, and magnetic skyrmions. The use of SANS for "hard" condensed matter science was the topic of a recent ORNL Neutron Scattering Division Program Development Workshop co-organized by Dr. Ken Littrell and Prof. Eskildsen.

\subsection{MARTIN GREVEN, UNIVERSITY OF MINNESOTA - 'NEUTRON SCATTERING STUDIES OF HIGH-TC CUPRATES IN HIGH MAGNETIC FIELDS.’}

Dr. Greven of the University of Minnesota began by noting that the cuprates remain a pivotal and profound materials physics challenge and that there remain several competing theories for understanding the nature of high-temperature superconductivity (HTS) in these complex oxides. Some of these theories can be tested using neutron scattering techniques in conjunction with a high magnetic field sample environment. As an example, two of these possible theories involve antiferromagnetic fluctuations and loop-current fluctuations. Theories such as the latter are tied to a quantum critical point that can only be revealed with high magnetic fields.

Neutron scattering measurements of the incommensurate magnetic response in the compound LSCO have already been found to be sensitive to applied magnetic field up to $14.5 \mathrm{~T}$. Transport (electric current) measurements have already helped to clarify parts of the cuprate phase diagram, by identifying metal-to-insulator phase transitions and quantum oscillations in high applied fields. For the compound YBCO, the HTS phase at the hole doping level of 0.12 is fully suppressed at $30 \mathrm{~T}$, pinching off regions of HTS at higher and lower doping levels. These transport results are complemented by the observation with synchrotron X-rays of charge-density-wave correlations in YBCO that increase as the magnetic field is increased from 0 to $17 \mathrm{~T}$.

Dr. Greven's group is uniquely able to produce sizable single crystals of the Hg1201 cuprate $\left(\mathrm{HgBa}_{2} \mathrm{CuO}_{4+\mathrm{d}}\right)$ for neutron scattering measurements, a compound whose crystalline structure is one of the simplest among the cuprates, and for which disorder effects appear to be minimal and the optimal superconducting transition temperature of nearly $100 \mathrm{~K}$ is particularly high. Initial magnetic neutron scattering experiments of the antiferromagnetic response already have been very revealing. Unlike other cuprates, which exhibit an X-shaped ("hourglass") magnetic dispersion, measurements of underdoped Hg1201 single crystals at the ARCS instrument at the SNS reveal a "Y" shape as a result of the high structural symmetry and minimal disorder effects. ${ }^{8}$ This work lays the foundation for future measurements of this model cuprate system as a function of doping, temperature and up to the highest attainable magnetic fields. By extending neutron scattering measurements to $30 \mathrm{~T}$ (or higher), and thus performing measurements that complement high-field transport and X-ray measurements of the charge degrees of freedom, potential theories that describe superconductivity in the cuprates can be narrowed down.

\subsection{DEAN MYLES, ORNL- 'DYNAMIC NUCLEAR POLARIZATION AND BIOLOGY.'}

Dr. Myles, leader of the Biology and Biomedical Sciences Group, Biology and Soft Matter Division, NScD, began with an overview of both the advantages and the challenges of locating hydrogen atoms in biological structures using neutron protein crystallography. The advantage derives from the critical role hydrogen atoms play in cell chemistry, including enzyme activity, hydrogen bond networks, 
photochemistry, DNA repair and replication, transport in membrane proteins and cell signaling. A critical step towards understanding these roles is the measurement of hydrogen locations in active parts of the protein. The IMAGINE instrument at HFIR, and the TOPAZ and MaNDI instruments at the SNS are all optimized for such applications, and Myles highlighted a recent tour-de-force was performed on RAS GTPase (a protein involved in cellular signal transduction) at IMAGINE. However, in most systems of interest, the incoherent background signal from hydrogen rapidly overwhelms the coherent elastic signal that is used to determine structure, severely limiting the resolution of the structural information that can be obtained in a protein crystallography experiment (i.e., the signal is buried in the background). In favorable cases, the scattering background can be reduced by substituting deuterium for hydrogen in the sample, but the approach requires custom synthesis and has had limited application.

Spin polarization of protons in the sample offers a potentially powerful solution to this problem. The neutron's interaction with hydrogen depends on the relative orientation of their spins. The origin of the large hydrogen incoherent scattering cross-section lies in the ordinarily unordered spin states of the neutrons and protons. Polarizing the neutron beam and the hydrogen nuclei in the sample drastically changes these cross-sections as shown in Figure 3.

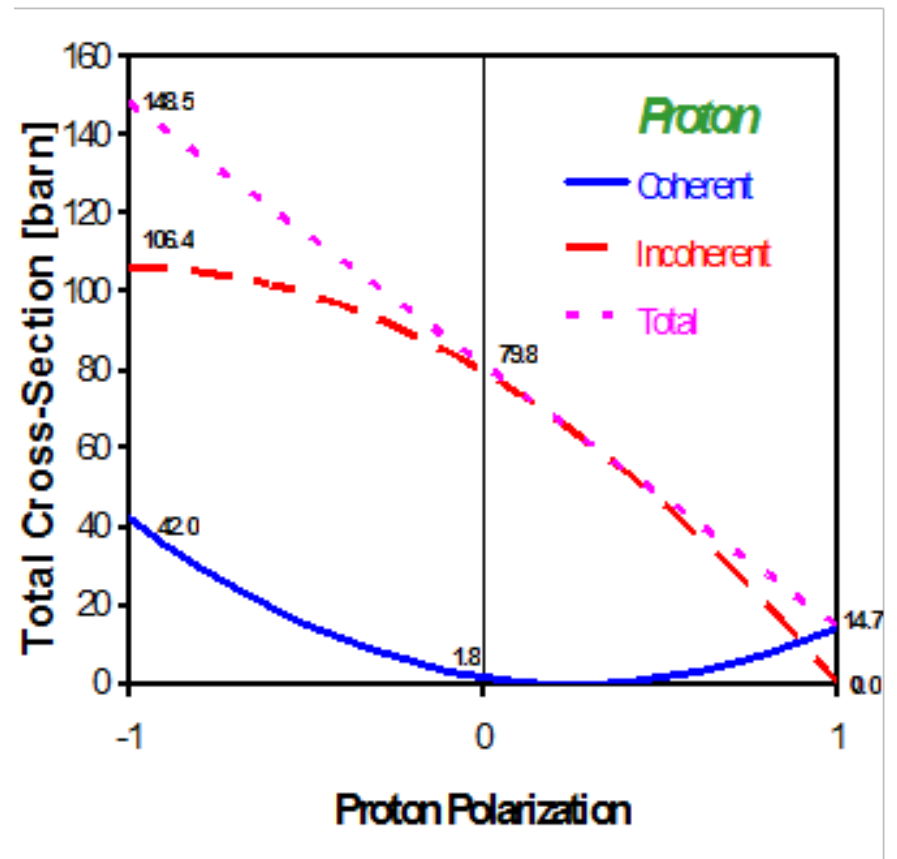

Figure 5. Coherent, incoherent and total scattering cross section of hydrogen as a function of the proton polarization for fully polarized neutrons. ${ }^{9}$ Figure is inspired by Fig. 1 of Ref. [10].

Dynamic nuclear polarization (DNP) offers an effective way to achieve a high degree (up to 99\%) of nuclear polarization in non-metallic samples. DNP is a process in which the large polarizations of electron spins in a strong magnetic field are partially transferred to nuclear spins by irradiation of electron paramagnetic resonance (EPR) transitions, resulting in large enhancements of nuclear spin polarizations. EPR is the resonant absorption of microwave radiation by paramagnetic ions or molecules with at least one unpaired electron spin in the presence of a static magnetic field. When using DNP to co-align and orient hydrogen nuclei, paramagnetic centers are added to the sample and polarizations are 'pumped' from easily polarized electron spins to nuclear ones using microwaves. When performing neutron diffraction on a hydrogen-rich crystal using DNP and a polarized neutron beam, the coherent scattering term is amplified by a near-order of magnitude and the incoherent background scattering terms is simultaneously suppressed. This technique both enhances the Bragg scattering and reduces the incoherent background. 
Nuclear polarization improves with colder crystal temperatures, requiring the use of a dilution refrigerator and careful tuning of microwave power to balance sample heating and saturation of the resonance. At temperatures of $<100 \mathrm{mK}$, nuclear spins are then 'frozen' in place for extended periods of time, enabling spin polarized neutron diffraction data to be collected. This technique holds promise for both protein crystallography and small angle neutron scattering, and feasibility has already been demonstrated for crystallography with small molecules. ${ }^{10}$

Research and development is now underway at ORNL to prepare an insert with a $2.5 \mathrm{~T}$ magnet, dilution refrigerator and microwave emitter for use at IMAGINE for protein crystallography. This insert is expected to provide an enhancement of $\sim 100$ in the signal to noise ratio, enabling for example the use of much smaller protein crystals. A dedicated DNP crystallography beam line on the STS called DYPOL has also been proposed, which will deliver a further $>50$-fold gain factor in instrument performance.

Direct nuclear polarization holds even further promise as significantly stronger magnets become available. Increasing the field to $>25 \mathrm{~T}$ allows sample temperatures of $\sim 100 \mathrm{mK}$, and increasing to $>60 \mathrm{~T}$ allows sample temperatures of $\sim 1 \mathrm{~K}$, removing the need for ${ }^{3} \mathrm{He}$ based cooling.

\subsection{VALERY KIRYUKHIN, RUTGERS UNIVERSITY- ‘ANISOTROPIC MAGNETS IN HIGH MAGNETIC FIELDS.’}

Dr. Kiryukhin, Rutgers University, before going into detail on his topic, began by reminding the attendees that magnetic field is one of a very few fundamental thermodynamic variables, together with temperature, pressure and electric field. He also noted that over $10 \%$ of recent Phys. Rev. Lett. and Phys. Rev. B publications report on effects of applied magnetic field, and that the field scale associated with room temperature is $100 \mathrm{~T}$. He then listed a set of example problems that he would like to see addressed using neutron scattering with high magnetic fields including:

- Investigation of modulations in HTS cuprates, with fields of 30-40 T. Fields of 70-100 T are needed to study the full range of phenomena.

- Magnetic field tuning of quantum criticality. Metamagnetic quantum critical points, heavy fermion materials. Magnetic quantum fluctuations in multiferroics.

The $\mathrm{BiFeO}_{3}$ system is introduced as the "dream" multiferroic, because it exhibits multiferroic behavior at room temperature, making it a functional material with possible commercial applications. Magnetic response observed by elastic neutron scattering has been tuned by applied electric field, and the cycloid nature of the Fe magnetic order has been observed with polarized neutron scattering. Separately, an applied magnetic field of only $20 \mathrm{~T}$ has been observed to unwind the cycloid, producing a room temperature weakly ferromagnetic ferroelectric. Neutron scattering studies at up to $20 \mathrm{~T}$ are required to better understand this multiferroic behavior.

With ferromagnets, optimum performance for electric motors and generators is found with a combination of high coercivity, $\mathrm{H}_{\mathrm{c}}$, and high saturation magnetization, $\mathrm{M}_{\mathrm{s}}$, leading to a high energy product. High magnetic coercivity refers to the large magnetic hysteresis, or the high applied field required to reverse or reorient the spins in the ferromagnet, and is usually associated with large magnetic anisotropy, and/or a domain boundary structure that enables suppressed domain nucleation or strong pinning of domain walls, and is often achieved via the inclusion of rare earth atoms. High saturation magnetization is associated with large magnetic moments per unit cell and is often associated with elements like iron. The strongest commercially available magnets, such as $\mathrm{Nd}_{2} \mathrm{Fe}_{14} \mathrm{~B}$ with $\mathrm{M}_{\mathrm{s}} \sim 1.4 \mathrm{~T}$ and $\mathrm{H}_{\mathrm{c}} \sim 0.25 \mathrm{~T}$, contain rare earths, but the United States is dependent on foreign sources and an expensive and volatile market for access to the rare earth elements (Nd, etc). Therefore, DOE has initiated the Advanced Research Projects Agency-Energy (ARPA-E) project "Rare Earth Alternatives in Critical Technologies" or REACT, to develop cost-effective alternatives to rare earth materials.

Two example hard ferromagnets that would benefit from neutron scattering studies at high field are $\mathrm{LuFe}_{2} \mathrm{O}_{4}$ and $\mathrm{Sr}_{3} \mathrm{NiIrO}_{6}$. $\mathrm{LuFe}_{2} \mathrm{O}_{4}$ (Lu plays no role in magnetism) has $\mathrm{H}_{\mathrm{c}} \sim 9$ T below the magnetic freezing temperature of $4 \mathrm{~K}$, and exhibits frozen Ising pancake-like magnetic domains. $\mathrm{Sr}_{3} \mathrm{NiIrO}_{6}$ has $\mathrm{H}_{\mathrm{c}} \sim 55 \mathrm{~T}$ at $4 \mathrm{~K}$, possibly due to the large spin orbit coupling of $\mathrm{Ir}(5 \mathrm{~d})$ and the associated large magnetic 
anisotropy, but the magnetic structures at high field are unknown. The final example system is a multiferroric system $\mathrm{Ca}_{3} \mathrm{CoMnO}_{6}$ with the same structure as the hard magnet $\mathrm{Sr}_{3} \mathrm{NiIrO}_{6}$. Magnetization measurements up to $30 \mathrm{~T}$ applied field show several plateaus that may be associated with different magnetic structures, but neutron diffraction has only been achieved up to $11 \mathrm{~T}$ applied field with this system. One important aspect of these proposed studies is that both high applied field and moderate applied field with wide solid angle acceptance are important for diffraction.

\subsection{COLLIN BROHOLM, JOHNS HOPKINS UNIVERSITY- 'THE HIGH FIELD FRONTIER AND QUANTUM MATTER.’}

Dr. Broholm presented measurements on several magnetic materials examining their excitations as a function of applied magnetic field. Quantum materials are particularly interesting to examine at high magnetic fields due to the many degrees of freedom that interact with one another to yield exotic excitations. One such excitation is known as a spinon. The spinon does not have sharp modes in its excitation spectrum; rather it has a continuum of excitations. Such continua are due to the spinon being a multiparticle excitation. These excitations have been observed in zero magnetic field and in modest magnetic fields using inelastic neutron scattering techniques. Larger magnetic fields would allow one to trace the evolution of the spinon excitations throughout their magnetic field and temperature dependent phase diagram.

$\mathrm{NiGa}_{2} \mathrm{~S}_{4}$ is a spin 1 triangular lattice antiferromagnet. At low temperatures, the magnetic moments in this compound do not exhibit long range order as in a classical antiferromagnet. Rather the geometrical frustration of the crystal lattice prevents the spins from ordering and only short correlation lengths are present. In order to understand the ground state of this geometrically frustrated system, $30 \mathrm{~T}$ magnetic fields would be required in a neutron scattering sample environment. Such large magnetic fields would be able to fully polarize the magnetic moments along the field direction. Diffraction and inelastic measurements would then be able to determine the nature of the magnetic interactions between the frustrated spins.

Quantum spin liquids are a new state of matter, and the continua excitations in these systems indicate that they also support spinon excitations. High magnetic field measurements are able to drive such systems through quantum critical points to examine the different length scales involved in the magnetic interactions. Applied magnetic fields also Zeeman split excitations by lifting the degeneracy present in the zero field Hamiltonian. This allows one to determine the degeneracy of the excitations.

Unconventional superconductors are another class of system where magnetism plays a critical role. High magnetic fields are necessary to reach the upper critical field. This will allow for the statics and dynamics of these materials to be examined throughout their phase diagrams in order to look for common behavior in this class of condensed matter system.

\subsection{MEIGAN ARONSON, BROOKHAVEN NATIONAL LABORATORY AND STONY BROOK UNIVERSITY -`OPPORTUNITIES FOR HIGH FIELD NEUTRON SCATTERING EXPERIMENTS IN QUANTUM CRITICAL SYSTEMS’}

Dr. Aronson of both Brookhaven National Laboratory and Stony Brook University, introduced quantum critical points (QCP's) as a conceptual lens through which one could approach many important systems. As temperature approaches $0 \mathrm{~K}$, no fluctuation modes have thermal energies and classical physics no longer applies, so behavior about phase transitions at $0 \mathrm{~K}$ can provide particularly important clues to understanding a system. The evidence for QCP's and similarities in phase diagrams for ferroelectrics and multiferroics, systems with charge density waves, heavy electron intermetallics, organic conductors and both cuprate and iron pnictide HTS's strongly suggest the importance of this approach. Perhaps it is even possible to map to a common phase diagram for correlated electron systems, with one axis being a tunable parameter and the other the degree of quantum fluctuations. Different correlated 
electron systems would have different degrees of quantum fluctuations due to dimensionality, frustration or dimerization. For metals, there is additional complexity due to a transition between two configurations with different sized Fermi surfaces.

Magnetic field is the best tuning parameter to use with neutron scattering, when it can suppress an ordered state by driving the phase transition temperature to $0 \mathrm{~K}$, due to limitations of other tuning parameters. The disorder introduced by compositional tuning can mask emergent phases, introduce new phases or modify quantum critical properties. Use of pressure as a parameter is limited by the pressure cell technology: only a few discrete pressure levels are normally achievable during a typical experiment, warm-up and cool-down consume valuable time due to the mass of the pressure cell, the limited solid angle and any surrounding material of the pressure cell affect data rate and Q range, and the upper pressures available are more limited for the larger samples needed for inelastic scattering. Applied magnetic field, in contrast, provides a continuously tunable parameter, and currently available magnets are already well matched to emergent energy scales for many material families. As higher fields become available, so will more energy scales compatible with other materials.

Quantum criticality may be possible for low dimensional ferromagnets. One possible QCP and Mott candidate is the quasi $2 \mathrm{D} \mathrm{YFe} \mathrm{Al}_{10}$. The temperature and field dependence of the measured susceptibility, specific heat and electrical resistivity can be mapped onto common curves, with critical exponents consistent with the $d=3 \mathrm{XY}$ model for quantum critical free energy; measurements were made with applied field up to $6 \mathrm{~T}$.

The dimerization often found in systems with a Shastry-Sutherland lattice (SSL) structure may be introducing quantum fluctuations in the $\mathrm{Yb}_{2} \mathrm{Pt}_{2} \mathrm{~Pb}$ system, and applied magnetic field up to $2.5 \mathrm{~T}$ can be used to tune from a phase where adjacent SSL layers are weakly coupled (mostly 2D), to one where spin ladders are weakly coupled via rungs in the SSL structure (mostly 1D). Magnetic structure at $0 \mathrm{~T}$ is striped in the SSL's with AF coupling / stacking between SSL planes. Magnetic excitations in the SSL plane are localized (flat) due to the orthogonality of the dimers, while spinon excitations between planes exhibit unusual broadening. One-dimensional physics seems to dominate excitations for $\omega<1.5 \mathrm{meV}$. A phase with modulated chains and Fermi liquid behavior is revealed at $2.5 \mathrm{~T}$, but between 1 and $2.3 \mathrm{~T}$ an interesting entropy-rich dome phase with parital magnetic order is formed at low temperature.

To explore similarly rich phase behaviors with copper based systems, with the higher copper magnetic moment, requires access to much higher magnetic field than is currently available at neutron scattering facilities. Dimerized spin chain systems like $\mathrm{TlCuCl}_{3}$ would benefit from fields up to $35 \mathrm{~T}$, SSL systems like $\mathrm{SrCu}_{2}\left(\mathrm{BO}_{3}\right)_{2}$ up to $60 \mathrm{~T}$ and layered spin dimers like $\mathrm{BaCuSi}_{2} \mathrm{O}_{6}$ up to $45 \mathrm{~T}$.

Magnet sample environment systems and neutron scattering instrumentation / configuration need to be considered together in order to maximize the potential for studying QCP systems. The goal is to bring the power of neutron scattering measurements to QCP materials with interesting behavior at high magnetic field, whose behavior to date has only been explored by electrical transport, magnetic susceptibility and specific heat measurements. Near QCP's there are no characteristic energy scales beyond temperature and magnetic field $\mathrm{H}$, where the relevant metrics become $\mathrm{E} / \mathrm{T}^{\alpha}$ and $\mathrm{g} \mu_{\mathrm{B}} \mathrm{H} / \mathrm{k}_{\mathrm{B}} \mathrm{T}$, both of which need to be explored at both very small and very high values (both high and low temperatures). Since quantum critical scattering is replaced at high temperature by other processes, both low temperatures and measurement of small excitation energies is required. Due to the mixed local and itinerant character of the response, a broad coverage of energies and wave vectors is also required.

\subsection{YOUNG LEE, MASSACHUSETTS INSTITUTE OF TECHNOLOGY-‘SCATTERING STUDIES OF QUANTUM MATERIALS: PROBING NEW STATES OF MAGNETISM’}

A Quantum Spin Liquid (QSL) is a state of matter that has only recently been observed in real systems. In a QSL, the atomic magnetic moments are strongly correlated, but do not order or freeze even as temperature goes to zero. Therefore, a QSL cannot be described by the broken translational or rotational symmetries as with normal ground states. Although the magnetic moments of a QSL do not order, they are entangled with each other over long ranges. This entanglement does not have a 
conventional order parameter, but may have a topological order that may have applications in quantum information. For example, there may be a conservation of the oddness or evenness of the number of singlets crossed when traversing the toroidal or cylindrical feature on a Fermi surface. Known candidates for QSL share the property of frustration.

An observable signature for QSL's is the presence of fractionalized excitations. The elementary excitations from the QSL ground state are spinons, which are spin-half excitations into which conventional spin wave excitations with $S=1$ (which can be directly measured via coherent inelastic neutron scattering) fractionalize, creating a continuum of spinon excitations. It is this signature that has been observed in some $1 \mathrm{D}$ systems like $\mathrm{KCuF}_{3}$, and most recently in the $2 \mathrm{D}$ spin-1/2 kagome-lattice antiferromagnet $\mathrm{ZnCu}_{3}(\mathrm{OD})_{6} \mathrm{Cl}_{2}$; also known as herbertsmithite. In herbertsmithite, even for $\mathrm{T}<<\mathrm{J}$, excitations are very diffuse and no spin gap is observed at any $Q$ point.

The reason why high magnetic fields could prove useful in understanding systems that demonstrate QSL, is because high field could suppress impurity states and ultimately the QSL state, enabling the study of what remains. By suppressing or freezing out impurity states, the measurement of fractionalized excitations becomes less controversial. Although the suppression of impurity states seems anticlimactic, it is in fact one of the more useful roles that magnetic fields can play in studying correlated electron systems. In the case of herbertsmithite, single crystals that have been successfully grown have $\sim 5 \%$ excess $\mathrm{Cu}^{2+}$ ions substituting for $\mathrm{Zn}^{2+}$ ions in interlayer sites, and these $\mathrm{Cu}^{2+}$ ions aren't really free impurities because they are coupled to neighboring kagome planes. Magnetic fields in excess of $10 \mathrm{~T}$ are expected to saturate these specific impurities.

At even higher field, the suppression of the QSL state is expected to lead to a ferromagnetic kagome system, enabling the determination of the spin Hamiltonian in this new phase, where measurements of both ferromagnetic dispersive modes and a flat band of excitations is predicted. By inducing this ferromagnetic order, the nature of the interactions could be clarified, aiding in understanding the QSL observed at lower field. More generally, high fields would be used to control the moment directions, creating gaps and new topological bands. 


\section{SUMMARY OF BREAKOUT SESSIONS}

\subsection{SHORT TERM NEEDS}

On the first day of the workshop a town-hall style discussion was held concerning the immediate needs for magnetic field sample environments at the SNS. We present here the topics and summaries of this discussion.

- The first step in enhancing the high magnetic field capabilities at the SNS is to purchase high magnetic field sample environments. The current highest operating magnet at the SNS has a maximum field of $5 \mathrm{~T}$. This is clearly inadequate given that the SNS is a flagship neutron facility. A $15 \mathrm{~T}$ vertical axis commercially available magnet should be pursued immediately.

- The ORNL neutron scattering facilities need to build up expertise in working with high magnetic field neutron scattering sample environments. This is especially true if the Zeemans project is to proceed further. An expert on low-temperature sample environments was recently hired into the sample environment group. This expertise has clearly made a significant improvement in the low-temperature capabilities of the sample environment group. One suggestion to build high magnetic field expertise is to promote collaborations on projects where these skills can be developed.

- There was discussion about pursuing a 25 T HTS uncompensated magnet. Zeemans is now being considered for the STS which would operate in 2022 at the earliest. A 25 Tesla HTS portable vertical split coil magnet would require 4 to 5 years of time to develop. Having both a Zeemans magnet and a $25 \mathrm{~T}$ magnet would be very useful for the user program. A 25 Tesla HTS magnet would require significant cooling and helium consumption would be a concern if the current is ramped frequently. Helium recovery would also be necessary given the amount of liquid helium such a magnet would consume. The HTS magnet would not operate in persistent mode; rather the current would be kept in the leads. The consensus was that the ORNL neutron scattering facilities are strongly encouraged that to implement such a facility particularly if the magnetic field capabilities are to expand.

- ORNL is encouraged to revisit the SNS stray field policy.

\subsection{STRATEGY TO HIGH MAGNETIC FIELDS AT SNS AND HFIR}

On the second day of the workshop a town-hall style discussion was held concerning the strategy to obtain high magnetic field sample environments or instruments at the SNS and HFIR neutron scattering facilities. We present here the topics and summaries of this discussion.

- Workshops are an important step in developing the magnetic field capabilities of SNS and HFIR. A large issue is making an excellent science case for the Zeemans instrument. Workshops and their reports are important to make this case.

- The potential for collaboration with university partners to expand the magnetic field capabilities of SNS and HFIR was discussed. University partners would be very useful in the proposal for the Zeemans beamline. In addition there are NSF funds available to university proposals for commercially available magnets. This would help to push the facilities beyond their current capabilities.

- For NSF, the involvement of the university community is critical and training and education are critical.

- ORNL's Laboratory Directed Research \& Development is an option to pursue funding for pulsed magnet capabilities beyond the Nojiri style magnets. Such a program also helps to develop the science case for a Zeemans instrument.

- The Zeemans instrument was discussed. One of the key questions that needs to be answered within approximately two years is what technology needs to be pursued for the magnet. The cost 
of a Zeemans instrument will be around $\$ 40 \mathrm{M}$. The cost of the STS would be of the order of $\$ 500 \mathrm{M}$. Because the Zeemans instrument is essential to the scientific case for the STS, it was proposed that Zeemans' cost be incorporated with the STS proposal. The NSF potential investment in a Zeemans scale project is likely to be relatively small.

- One strategy is to push for the "next generation Nojiri" pulsed magnet. This could leverage some in-kind contributions from NHMFL at the Los Alamos National Laboratory, and potential resources from the National Science Foundation (NSF).

- $\quad$ Sample size was discussed. The SNS should perform a proof of principle measurement on a well characterized magnetic system such as $\mathrm{SrCu}\left(\mathrm{BO}_{3}\right)_{2}$. The experiment would be one on a cold neutron chopper spectrometer such as CNCS at the SNS. The measurement would start with a single crystal and continue to cut it in half to see how small a sample one can measure with a reasonable signal to noise ratio. This information is needed in order to make estimates of feasibility for magnets designed for use with small sample sizes.

- Some of the options for pulsed magnets were discussed - split coil, horizontal bore, conical bore, and samples located at the edge of a horizontal bore. The technology needs to develop toward a $30 \mathrm{~T}, 1 \mathrm{~Hz}$ pulse frequency. This was noted as being a potential route achievable by mid-2016.

- It was noted that, in principle, a HTS magnet would be easier to fabricate than a series connected hybrid. A HTS magnet may arrive more quickly than the Zeemans instrument. Such a magnet would be designed for use with existing instruments. A split coil magnet in the 20-25 T field range would be comparable in size to FAT SAM, but without compensating coils.

- A list of magnet candidates for development includes:

o Pulsed split vertical coil magnet $>25 \mathrm{~T}$ - requires $1 \mathrm{~Hz}$ or faster for inelastic, but would also expand capabilities for neutron diffraction.

o Standardize the use of pulsed horizontal bore 'Nojiri style' magnet - This includes examining behavior with sample located downstream of magnet center in order to increase solid angle acceptance.

o HTSC split coil 20-25 T magnet 


\section{REFERENCES}

${ }^{1}$ High Magnetic Field Science and Its Application in the United States: Current Status and Future Directions, (The National Academies Press, Washington, D. C. 2013).

${ }^{2}$ Workshop on Quantum Condensed Matter, Report, University of Berkley, December, 2013.

${ }^{3}$ M. Steiner, D. A. Tennant and P. Smeibidl, Journal of Physics: Conference Series 51, 470 (2006).

${ }^{4}$ P. Smeibidl, A. Tennant, H. Ehmler and M. Bird, Journal of Low Temperature Physics 159, 402 (2010).

${ }^{5}$ H. Nojiri et al., Physical Review Letters 106, 237202 (2012). Pulsed field experimental data was acquired at the SEQUOIA instrument at SNS.

${ }^{6}$ Z. Islam et al., Review of Scientific Instruments 83, 035101 (2012).

${ }^{7}$ M. D. Bird, H. Bai, S. Bole, J. Chen, I. R. Dixon, H. Ehmler, A. V. Gavrilin, T. A. Painter, P. Smeibidl, J. Toth, H. Weijers, T. Xu and Y. Zhai, IEEE Transactions on Applied Superconductivity 19, 1612 (2009).

${ }^{8}$ M. K. Chan et al., arXiv: 1402.4517.

${ }^{9}$ J. K. Zhao et al., (2013).

${ }^{10}$ F. M. Piegsa, M. Karlsson, B. van den Brandt, C. J. Carlile, E. M. Forgan, P. Hautle, J. A. Konter, G. J. McIntyre and O. Zimmer, Journal of Applied Crystallography 46, 30 (2013). 
APPENDIX A. PARTICIPANTS, PRESENTATIONS AND AGENDA 


\section{APPENDIX A.1 PARTICIPANTS}

Table 2. Table of workshop participants.

\begin{tabular}{|c|c|c|}
\hline Name & Institution & Email address \\
\hline Meigan Aronson & Stony Brook University & maronson@bnl.gov \\
\hline Mark Bird & Florida State University & bird@magnet.fsu.edu \\
\hline Greg Boebinger & Florida State University & gsb@magnet.fsu.edu \\
\hline Collin Broholm & Johns Hopkins University & broholm@pha.jhu.edu \\
\hline Roy Cutler & ORNL & cutlerri@ornl.gov \\
\hline John DiTusa & Louisiana State University & ditusa@phys.lsu.edu \\
\hline Morten Eskildsen & University of Notre Dame & eskildsen@nd.edu \\
\hline Garrett Granroth & ORNL & granrothge@ornl.gov \\
\hline Martin Greven & University of Minnesota & greven@physics.umn.edu \\
\hline Ken Herwig & ORNL & herwigkw@ornl.gov \\
\hline Valery Kiryukhin & Rutgers University & vkir@physics.rutgers.edu \\
\hline Young Lee & Massachusetts Institution of Technology & younglee@mit.edu \\
\hline Mark Lumsden & ORNL & lumsdenmd@ornl.gov \\
\hline Gary Lynn & ORNL & lynngw@ornl.gov \\
\hline Masaaki Matsuda & ORNL & matsudam@ornl.gov \\
\hline Robert McQueeney & ORNL & mcqueeneyrj@ornl.gov \\
\hline Mark Meisel & University of Florida & meisel@phys. Ufl.edu \\
\hline Chuk Mielke & Los Alamos National Laboratory & cmielke@lanl.gov \\
\hline Martin Mourigal & Johns Hopkins University & mourigal@pha.jhu.edu \\
\hline Dean Myles & ORNL & mylesda@orn.gov \\
\hline Stephen Nagler & ORNL & naglerse@ornl.gov \\
\hline Yasuo Narumi & Tohoku University & narumi@imr.tohoku.ac.jp \\
\hline Ward Plummer & Louisiana State University & wplummer@phys.lsu.edu \\
\hline Peter Smeibidl & Helmholtz-Zentrum Berlin & peter.smeibidl@helmholtz-berlin.de \\
\hline Matthew Stone & ORNL & stonemb@ornl.gov \\
\hline Allan Tennant & ORNL & tennantda@ornl.gov \\
\hline Guebre X. Tessema & National Science Foundation & gtessema@nsf.gov \\
\hline John Tranquada & Brookhaven National Laboratory & jtran@bnl.gov \\
\hline Barry Winn & ORNL & winnbl@ornl.gov \\
\hline
\end{tabular}




\section{APPENDIX A.2 PRESENTATIONS}

Table 3. Table of workshop presentations.

\begin{tabular}{|l|l|l|}
\hline Name & Institution & Title \\
\hline Meigan Aronson & Stony Brook University & High Field Opportunities in Correlated Matter \\
\hline Mark Bird & Florida State University & DC Magnet Technologies Relevant for Neutron Scattering \\
\hline Greg Boebinger & Florida State University & Probing Quantum Matter: Neutrons and High Magnetic Fields \\
\hline Collin Broholm & Johns Hopkins University & The High Field Frontier and Quantum Magnetism \\
\hline Morten Eskildsen & University of Notre Dame & $\begin{array}{l}\text { Vortex Lattice Metastability and Power Law Dynamics in } \\
\text { MgB } 2\end{array}$ \\
\hline Garrett Granroth & ORNL & Zeemans: A Dedicated High Field Beam Line For the SNS \\
\hline Martin Greven & University of Minnesota & $\begin{array}{l}\text { Neutron Scattering Studies of High-Tc Cuprates in High } \\
\text { Magnetic Fields }\end{array}$ \\
\hline Ken Herwig & ORNL & Challenges and Opportunities of the 2 ${ }^{\text {nd }}$ Target Station \\
\hline Valery Kiryukhin & Rutgers University & Anisotropic Magnets in High Magnetic Fields \\
\hline Young Lee & $\begin{array}{l}\text { Massachusetts Institution } \\
\text { of Technology }\end{array}$ & $\begin{array}{l}\text { Scattering Studies of Quantum Materials: Probing New States } \\
\text { of Magnetism }\end{array}$ \\
\hline Gary Lynn & ORNL & $\begin{array}{l}\text { Magnet Sample Environments at ORNL: Status and Near- } \\
\text { Term Plan }\end{array}$ \\
\hline Thomas Mason & ORNL & Prospects of STS \\
\hline Chuck Mielke & $\begin{array}{l}\text { Los Alamos National } \\
\text { Laboratory }\end{array}$ & Magnet Technologies for Neutron Scattering \\
\hline Dean Myles & ORNL & Dynamic Nuclear Polarization and Biology \\
\hline Stephen Nagler & ORNL & Neutron Scattering and Condensed Matter at ORNL \\
\hline Yasuo Narumi & Tohoku University & $\begin{array}{l}\text { 40 T Pulsed Magnetic Fields Neutron Diffraction-Recent } \\
\text { Progress and Applications }\end{array}$ \\
\hline Peter Smeibidl & $\begin{array}{l}\text { Helmholtz-Zentrum } \\
\text { Berlin } \\
\text { Place At the HZB }\end{array}$ \\
\hline Allan Tennant & ORNL & Welcome and Charge \\
\hline John Tranquada & $\begin{array}{l}\text { Brookhaven National } \\
\text { Laboratory }\end{array}$ & $\begin{array}{l}\text { Probing Intertwined Orders in Cuprate Superconductors with } \\
\text { High Magnetic Fields }\end{array}$ \\
\hline
\end{tabular}




\section{APPENDIX A.3 WORKSHOP AGENDA}

\section{OAK RIDGE}

National Laboratory

\section{AGENDA}

MANAGED BY UT-BATTELLE FOR THE US DEPARTMENT OF ENERGY

\section{The Neutron Scattering in High Magnetic Fields W orkshop September 4-5, 2014}

Thursday, September 4, 2014

Building: 8600, Room C156

\begin{tabular}{|c|c|c|}
\hline Time & Event & Lead \\
\hline $8: 30-9: 00 \mathrm{am}$ & Registration & \\
\hline $9: 00-9: 30 \mathrm{am}$ & Welcome and Charge & Dr. Alan Tennant \\
\hline $9: 30-10: 00 \mathrm{am}$ & Neutron Scattering and Condensed Matter at ORNL & Dr. Stephen Nagler \\
\hline $10: 00-10: 30 \mathrm{am}$ & Probing Quantum Matter: Neutrons and High Magnetic Fields & Dr. Greg Boebinger \\
\hline $10: 30-10: 45 \mathrm{am}$ & Distribution of National Academy report & Dr. Matt Stone \\
\hline $10: 45-11: 00 \mathrm{am}$ & Morning Break refreshments served & \\
\hline $11: 00-11: 30 \mathrm{am}$ & Magnet Technologies for Neutron Scattering & Dr. Chuck Mielke \\
\hline $11: 30-12: 00 \mathrm{pm}$ & $\begin{array}{l}\text { Magnet Sample Environments at ORNL: Status and Near-Term } \\
\text { Plan }\end{array}$ & Dr. Gary Lynn \\
\hline $12: 00-1: 00 \mathrm{pm}$ & Working Lunch: Near-Term Magnetic Field Needs & Dr. Gary Lynn \\
\hline $1: 00-1: 30 \mathrm{pm}$ & $\begin{array}{l}\text { High Field Capabilities, Opportunities, and Challenges in Place } \\
\text { At the HZB }\end{array}$ & Dr. Peter Smeibidl \\
\hline $1: 30-2: 00 \mathrm{pm}$ & $\begin{array}{l}40 \mathrm{~T} \text { Pulsed Magnetic Fields Neutron Diffraction-Recent } \\
\text { Progress and Applications }\end{array}$ & Dr. Yasuo Narumi \\
\hline $2: 00-2: 30 \mathrm{pm}$ & DC Magnet Technologies Relevant for Neutron Scattering & Dr. Mark Bird \\
\hline $2: 30-2: 45 \mathrm{pm}$ & Afternoon Break refreshments served & \\
\hline $2: 45-3: 00 \mathrm{pm}$ & Discussion: Near-Term Magnetic Field Needs continued. & Dr. Matthew Stone \\
\hline $3: 00-3: 30 \mathrm{pm}$ & Challenges and Opportunities of the $2^{\text {nd }}$ Target Station & Dr. Kenneth Herwig \\
\hline $3: 30-4: 00 \mathrm{pm}$ & Zeemans: A Dedicated High Field Beam Line For the SNS. & Dr. Garrett Granroth \\
\hline 4:00-5:30pm & $\begin{array}{l}\text { Discussion: Long Term Vision, Including Beam Line Dedicated } \\
\text { High-Field Magnets, at the SNS and HFIR. Please Refer to } \\
\text { National Academy of Sciences report }\end{array}$ & Dr. Matt Stone \\
\hline $6: 00 \mathrm{pm}$ & $\begin{array}{l}\text { Working Dinner: Calhoun's Lenoir City Presentation: } \\
\text { Prospects of STS }\end{array}$ & Dr. Thom Mason \\
\hline
\end{tabular}


The Neutron Scattering in High Magnetic

Fields W orkshop

September 4-5, 2014

Friday, September 5, 2014

Building: 8600, Room C156

\begin{tabular}{|l|l|l|}
\hline Time & Event & Lead \\
\hline 9:00-9:30am & $\begin{array}{l}\text { Probing Intertwined Orders in Cuprate Superconductors with } \\
\text { High Magnetic Fields }\end{array}$ & Dr. John Tranquada \\
\hline 9:30-10:00am & Vortex Lattice Metastability and Power Law Dynamics in MgB 2 & Dr. Morten Eskildsen \\
\hline 10:00-10:30am & $\begin{array}{l}\text { Neutron Scattering Studies of High-Tc Cuprates in High } \\
\text { Magnetic Fields }\end{array}$ & Dr. Martin Greven \\
\hline 10:30-10:45am & Group photograph & \\
\hline 10:45-11:00am & Morning Break refreshments served & \\
\hline 11:00-11:30am & Dynamic Nuclear Polarization and Biology & Dr. Dean Myles \\
\hline 11:30-12:00pm & Anisotropic Magnets in High Magnetic Fields & Dr. Valery Kiryukhin \\
\hline 12:00-1:00pm & Working Lunch: Strategy for magnetic fields beyond 15 Tesla. & Dr. Barry Winn \\
\hline 1:00-1:30pm & Title-TBD & Dr. Collin Broholm \\
\hline 1:30-2:00pm & High Field Opportunities in Correlated Matter & Dr. Meigan Aronson \\
\hline 2:00-2:30pm & $\begin{array}{l}\text { Scattering Studies of Quantum Materials: Probing New States of } \\
\text { Magnetism }\end{array}$ & Dr. Young Lee \\
\hline 2:30-2:45pm & Afternoon Break refreshments served & Dr. Mark Lumsden \\
\hline 2:45-5:00pm & Discussion: Strategy for magnetic fields beyond 15 Tesla and & \\
\hline
\end{tabular}




\section{APPENDIX B. MAGNETIC FIELD SAMPLE ENVIRONMENTS AVAILABLE AT NEUTRON SCATTERING FACILITIES}

A survey of the websites of the higher flux neutron scattering facilities was performed to determine what magnetic field sample environments are available. These facilities are listed in Table 2. The facilities examined included reactor based, continuous spallation, and pulsed spallation neutron sources. Horizontal and Vertical field magnets were found to be in the user program at all of the facilities. In addition, the SNS, ISIS, ILL and MLF operate at least one pulsed magnet system with maximum fields of 30, 40, 40, and $50 \mathrm{~T}$ respectively.

Table 4. List of neutron scattering facilities worldwide with a user program and operation of magnetic field sample environments.

\begin{tabular}{|c|c|c|c|}
\hline Facility & Abbreviation & Source and power & Sample environment website \\
\hline $\begin{array}{l}\text { High Flux Isotope Reactor } \\
\text { at ORNL, USA }\end{array}$ & HFIR & Reactor, $80 \mathrm{MW}$ & http://neutrons.ornl.gov/equipment/?facility=HFIR \\
\hline $\begin{array}{l}\text { Canadian Neutron Beam } \\
\text { Centre, Canada }\end{array}$ & CNBC & Reactor, $120 \mathrm{MW}$ & http://www.aecl.ca/site/media/Parent/Spectrometers_Eng.pdf \\
\hline $\begin{array}{l}\text { Institut Laue-Langevin, } \\
\text { France }\end{array}$ & ILL & Reactor, $68 \mathrm{MW}$ & $\begin{array}{l}\text { http://www.ill.eu/instruments-support/sample- } \\
\text { environment/equipment/low-temperatures/ }\end{array}$ \\
\hline $\begin{array}{l}\text { The OPAL research } \\
\text { reactor at the Australian } \\
\text { Nuclear Science and } \\
\text { Technology Organisation, } \\
\text { Australia }\end{array}$ & ANSTO & Reactor, $20 \mathrm{MW}$ & $\begin{array}{l}\text { http://www.ansto.gov.au/ResearchHub/Bragg/Facilities/Sample } \\
\text { Environments/index.htm }\end{array}$ \\
\hline $\begin{array}{l}\text { The FRM-II reactor at the } \\
\text { Heinz Maier-Leibnitz } \\
\text { Zentrum, Germany }\end{array}$ & FRM-II & Reactor, $20 \mathrm{MW}$ & http://www.mlz-garching.de/se \\
\hline $\begin{array}{l}\text { NIST Center for Neutron } \\
\text { Research, USA }\end{array}$ & NIST & Reactor, $20 \mathrm{MW}$ & http://www.ncnr.nist.gov/equipment/ancequip.html \\
\hline $\begin{array}{l}\text { The BER-II reactor at the } \\
\text { Helmholtz Zentrum } \\
\text { Berlin, Germany }\end{array}$ & HZB & Reactor, $20 \mathrm{MW}$ & $\begin{array}{l}\text { http://www.helmholtz-berlin.de/user/experimental- } \\
\text { infrastructures/sample-environment/index_en.html }\end{array}$ \\
\hline $\begin{array}{l}\text { The Swiss Spallation } \\
\text { Neutron Source at the Paul } \\
\text { Scherrer Institute, } \\
\text { Switzerland }\end{array}$ & SINQ & $\begin{array}{l}\text { Continuous } \\
\text { Spallation, 20 MW } \\
\text { reactor equivalent }\end{array}$ & http://lns00.psi.ch/sinqwiki/Wiki.jsp?page=SampleEnvironment \\
\hline $\begin{array}{l}\text { Spallation Neutron Source } \\
\text { at ORNL, USA }\end{array}$ & SNS & $\begin{array}{l}\text { Pulsed Spallation, } 1.4 \\
\text { MW }\end{array}$ & http://neutrons.ornl.gov/instruments/SNS/sample/ \\
\hline $\begin{array}{l}\text { The ISIS neutron source at } \\
\text { the Rutherford Appleton } \\
\text { Laboratory, UK }\end{array}$ & ISIS & $\begin{array}{l}\text { Pulsed Spallation, } \\
0.18 \mathrm{MW}\end{array}$ & $\begin{array}{l}\text { http://www.isis.stfc.ac.uk/sample-environment/sample- } \\
\text { environment-7130.html }\end{array}$ \\
\hline $\begin{array}{l}\text { The Materials and Life } \\
\text { Science Experimental } \\
\text { Facility at J-PARC, Japan }\end{array}$ & MLF & $\begin{array}{l}\text { Pulsed Spallation, } 0.3 \\
\text { MW }\end{array}$ & $\begin{array}{l}\text { http://www.j- } \\
\text { parc.jp/researcher/MatLife/en/instrumentation/ns.html }\end{array}$ \\
\hline $\begin{array}{l}\text { The Lujan Center at Los } \\
\text { Alamos National } \\
\text { Laboratory, USA }\end{array}$ & Lujan & $\begin{array}{l}\text { Pulsed Spallation, } 0.1 \\
\text { MW }\end{array}$ & http://lansce.lanl.gov/lujan/ancequip.shtml \\
\hline
\end{tabular}

Figure 4 was produced from the data available at the neutron source websites listed in Table 4. Individual magnet sample environments available to users are plotted as boxes contributing to the total magnetic field capability for each of the neutron sources. Boxes are color coded according to the type of magnet available. Pulsed magnets are not included in this comparison. Magnets that are beamline specific are included in this comparison of facilities, and are often located at magnetism reflectometer instruments. Although frequency of use of these magnets was emphasized as an important factor, due to 
the limited information available this metric is not included. The magnetic field sample environments shown for the ORNL facilities represent a current 'snap-shot' of the working magnetic field sample environments.

To become competitive with other high flux neutron sources, the workshop has recommended the rapid acquisition of two commercially available magnet sample environments. These are shown as open rectangles in the figure. One is an $11 \mathrm{~T}$ horizontal field magnet for the HFIR, and the other is a $15 \mathrm{~T}$ vertical field magnet for the SNS. The Zeemans instrument was also recommended to be pursued by the workshop. This is illustrated as a 40 Tesla magnet for the second target station (STS) of the SNS. An $8 \mathrm{~T}$ vertical field magnet is in procurement stage for the SNS facility as shown in the blue shaded rectangle in Figure 4 in the SNS column.

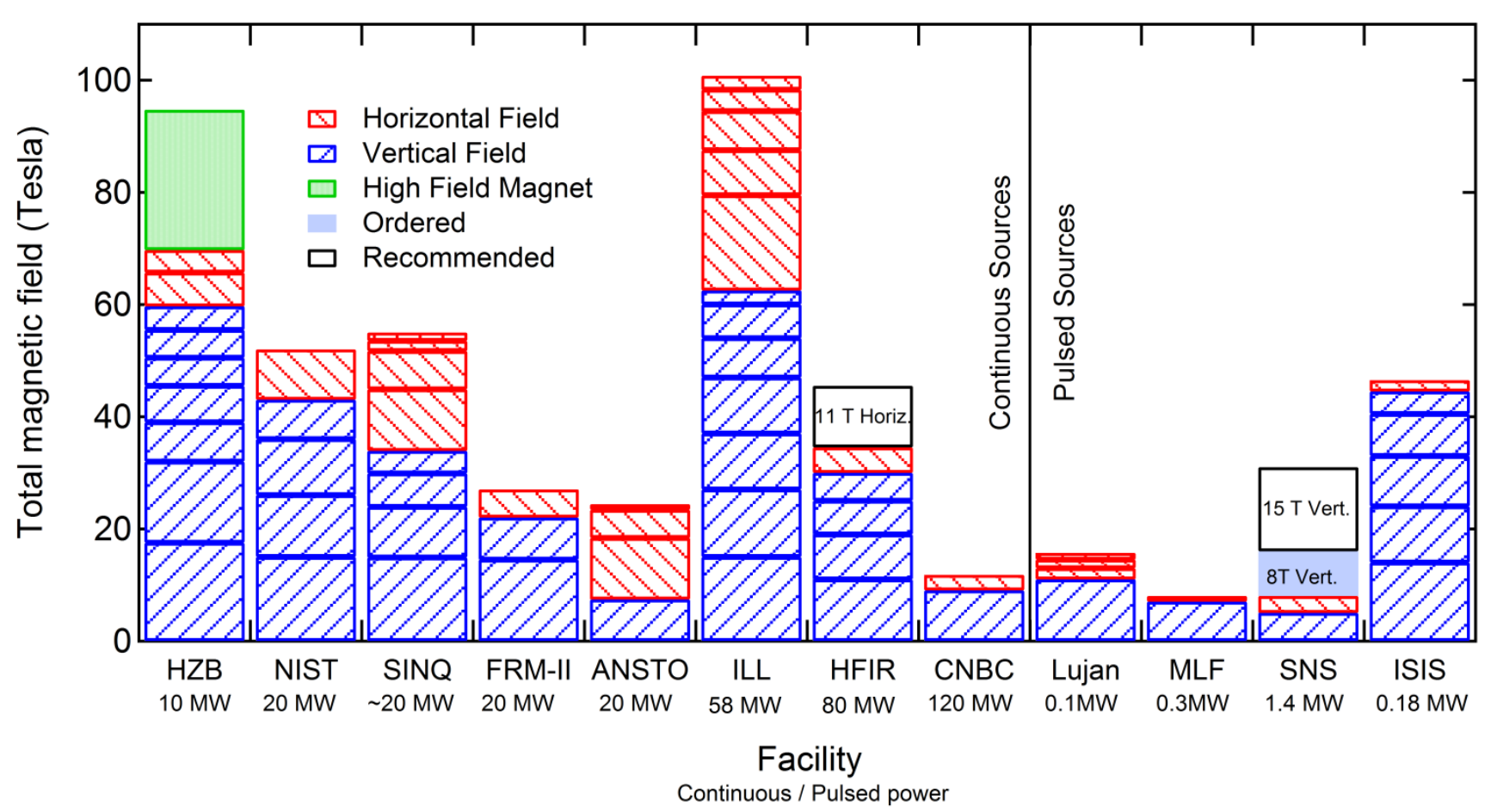

Figure 6. Total magnetic field sample environment capability available at several neutron scattering facilities. Continuous sources are shown in the left columns, and pulsed sources are depicted on the right. The Facility abbreviations are described in Table 4. Red hatched boxes represent individual horizontal field magnet sample environments. The magnetic field sample environments shown for the ORNL facilities represent a current 'snapshot' of the working magnetic field sample environments. Blue hatched boxes represent vertical field magnet sample environments. Sample environments which are dedicated to individual beamlines are also shown in this figure. The green shaded box represents the 25 T high field magnet, which will come online at the HZB in late 2014 or 2015. The grey light blue shaded box for the SNS represents an $8 \mathrm{~T}$ wide split vertical field magnet which has been ordered, and will be delivered to the SNS in late 2014 or early 2015. Pulsed magnets are not shown in this figure. Open black rectangles represent commercial magnets recommended by the consensus of the workshop attendees for purchase. The Zeemans instrument was also recommended by the workshop but is not illustrated in the figure as it would most likely be built as part of the second target station for SNS. 